\title{
Unpolarized isovector quark distribution function from lattice QCD: A systematic analysis of renormalization and matching
}

\author{
Yu-Sheng Liu, ${ }^{1}$ Jiunn-Wei Chen, ${ }^{2,3}$ Yi-Kai Huo, ${ }^{4,5}$ Luchang Jin, ${ }^{6,7}$ Maximilian Schlemmer, ${ }^{8}$ Andreas Schäfer, ${ }^{8}$ \\ Peng Sun, ${ }^{9,}$ Wei Wang, ${ }^{4, \dagger}$ Yi-Bo Yang $\odot,{ }^{10,11}$ Jian-Hui Zhang, ${ }^{8,12}$ Qi-An Zhang, ${ }^{1}$ \\ Kuan Zhang, ${ }^{13,11}$ and Yong Zhao, ${ }^{3,14}$ \\ (Lattice Parton Collaboration) \\ ${ }^{1}$ Tsung-Dao Lee Institute, Shanghai Jiao Tong University, Shanghai 200240, China \\ ${ }^{2}$ Department of Physics, Center for Theoretical Physics, and Leung Center for Cosmology and Particle \\ Astrophysics, National Taiwan University, Taipei 106, Taiwan \\ ${ }^{3}$ Center for Theoretical Physics, Massachusetts Institute of Technology, \\ Cambridge, Massachusetts 02139, USA \\ ${ }^{4} I N P A C$, SKLPPC, MOE KLPPC, School of Physics and Astronomy, Shanghai Jiao Tong University, \\ Shanghai 200240, China \\ ${ }^{5}$ Zhiyuan College, Shanghai Jiao Tong University, Shanghai 200240, China \\ ${ }^{6}$ Physics Department, University of Connecticut, Storrs, Connecticut 06269-3046, USA \\ ${ }^{7}$ RIKEN BNL Research Center, Brookhaven National Laboratory, Upton, New York 11973, USA \\ ${ }^{8}$ Institut für Theoretische Physik, Universität Regensburg, D-93040 Regensburg, Germany \\ ${ }^{9}$ Nanjing Normal University, Nanjing, Jiangsu 210023, China \\ ${ }^{10}$ Department of Physics and Astronomy, Michigan State University, East Lansing, Michigan 48824, USA \\ ${ }^{11}$ CAS Key Laboratory of Theoretical Physics, Institute of Theoretical Physics, \\ Chinese Academy of Sciences, Beijing 100190, China \\ ${ }^{12}$ Center of Advanced Quantum Studies, Department of Physics, Beijing Normal University, \\ Beijing 100875, China \\ ${ }^{13}$ University of Chinese Academy of Sciences, School of Physical Sciences, Beijing 100049, China \\ ${ }^{14}$ Physics Department, Brookhaven National Laboratory, Building 510A, Upton, New York 11973, USA
}

(Received 22 October 2018; revised manuscript received 4 December 2019; accepted 27 January 2020; published 18 February 2020)

\begin{abstract}
We present a detailed lattice QCD study of the unpolarized isovector quark parton distribution function (PDF) using a large-momentum effective theory framework. We choose a quasi-PDF defined by a spatial correlator which is free from mixing with other operators of the same dimension. In the lattice simulation, we use a Gaussian-momentum-smeared source at $M_{\pi}=356 \mathrm{MeV}$ and $P_{z} \in\{1.8,2.3\} \mathrm{GeV}$. To control the systematics associated with the excited states, we explore five different source-sink separations. The nonperturbative renormalization is conducted in a regularization-independent momentum subtraction scheme, and the matching between the renormalized quasi-PDF and $\overline{\mathrm{MS}}$ PDF is calculated based on perturbative QCD up to one-loop order. Systematic errors due to renormalization and perturbative matching are also analyzed in detail. Our results for light-cone PDF are in reasonable agreement with the latest phenomenological analysis.
\end{abstract}

DOI: 10.1103/PhysRevD.101.034020

\footnotetext{
${ }^{*}$ Corresponding author. 06260@njnu.edu.cn

Corresponding author. wei.wang@sjtu.edu.cn
}

Published by the American Physical Society under the terms of the Creative Commons Attribution 4.0 International license. Further distribution of this work must maintain attribution to the author(s) and the published article's title, journal citation, and DOI. Funded by SCOAP.

\section{INTRODUCTION}

Parton distribution functions (PDFs) of nucleons are not only important quantities characterizing the internal hadron structures but are also key ingredients to make predictions for high-energy scattering processes [1-3]. Thus calculating PDFs from first principles has been a holy grail in nuclear and particle physics. Since PDFs are embedded with the low-energy quark and gluon degrees of freedom in 
the hadron, they involve infrared (IR) dynamics of strong interactions and can only be determined by nonperturbative methods such as lattice QCD.

Within QCD factorization [4], the quark PDF is defined as

$q(x, \mu) \equiv \int \frac{d \xi^{-}}{4 \pi} e^{-i x P^{+} \xi^{-}}\left\langle P\left|\bar{\psi}\left(\xi^{-}\right) \gamma^{+} U\left(\xi^{-}, 0\right) \psi(0)\right| P\right\rangle$,

where $|P\rangle$ denotes the nucleon state with momentum $P_{\mu}=\left(P_{t}, 0,0, P_{z}\right) . x$ is the quark momentum fraction, $\mu$ is the renormalization scale in the $\overline{\mathrm{MS}}$ scheme. $\xi^{ \pm}=$ $(t \pm z) / \sqrt{2}$ are the light-cone coordinates. The lightlike Wilson line is introduced to maintain the gauge invariance:

$$
U\left(\xi^{-}, 0\right)=P \exp \left(-i g \int_{0}^{\xi^{-}} d \eta^{-} A^{+}\left(\eta^{-}\right)\right)
$$

PDFs are defined with light-cone coordinates, but the lattice simulation can only be conducted in Euclidean space with no proper treatment for light-cone quantities, which involves real time. Thus simulating PDFs on a Euclidean lattice is an extremely difficult task. Early studies based on operator product expansion were only able to derive the lowest few moments of the PDFs [5-8].

Recently, a novel approach that allows to directly access the $x$ dependence of PDFs from lattice QCD was proposed in Ref. [9], now formulated as large-momentum effective theory (LaMET) [10]. Within this framework, one can extract PDFs - as well as other light-cone quantities-from the correlations of certain static operators in a nucleon state. On the one hand, the static correlations, often referred to as quasiobservables, can be directly calculated on a Euclidean lattice and depend dynamically on the nucleon momentum. On the other hand, at large momentum, the quasiobservables can be factorized into the parton observable and a perturbative matching coefficient, up to corrections suppressed by powers of the large nucleon momentum. Equating the results from the two sides provides a straightforward way to determine the light-cone PDFs.

To calculate the quark PDF in LaMET, one starts with a "quasi-PDF" which is defined as an equal-time correlation of quarks along the $z$ direction [9]:

$$
\tilde{q}_{\Gamma}\left(x, P_{z}\right) \equiv \int_{-\infty}^{\infty} \frac{d z}{4 \pi} e^{i x P_{z} z}\left\langle P\left|O_{\Gamma}(z)\right| P\right\rangle .
$$

In the above, $O_{\Gamma}(z)=\bar{\psi}(z) \Gamma U(z, 0) \psi(0)$ with $\Gamma=\gamma^{z}$ or $\Gamma=\gamma^{t}$, and the spacelike Wilson line is

$$
U(z, 0)=P \exp \left(-i g \int_{0}^{z} d z^{\prime} A_{z}\left(z^{\prime}\right)\right) .
$$

For finite but large momentum $P_{z}, \tilde{q}\left(x, P_{z}\right)$ has support in $-\infty<x<\infty$. Unlike the light-cone PDF that is boost invariant, the quasi-PDF has a nontrivial dependence on the nucleon momentum $P_{z}$. After renormalizing the quasi-PDF in a scheme such as the regularization-independent momentum subtraction (RI/MOM) scheme, one can match the renormalized quasi-PDF to the $\overline{\mathrm{MS}} \mathrm{PDF}$ through the factorization theorem [9-14]:

$$
\begin{aligned}
\tilde{q}\left(x, P_{z}, p_{z}^{R}, \mu_{R}\right)= & \int_{-1}^{1} \frac{d y}{|y|} C\left(\frac{x}{y}, r, \frac{y P_{z}}{\mu}, \frac{y P_{z}}{p_{z}^{R}}\right) q(y, \mu) \\
& +\mathcal{O}\left(\frac{M^{2}}{P_{z}^{2}}, \frac{\Lambda_{\mathrm{QCD}}^{2}}{x^{2} P_{z}^{2}}\right),
\end{aligned}
$$

where $p_{z}^{R}$ and $\mu_{R}$ are introduced in the RI/MOM scheme. $p_{z}^{R}$ is the momentum of the involved parton and $\mu_{R}$ is renormalization scale. $r=\mu_{R}^{2} /\left(p_{z}^{R}\right)^{2}, C$ is the perturbative matching coefficient, and $\mathcal{O}\left(M^{2} / P_{z}^{2}, \Lambda_{\mathrm{QCD}}^{2} / x^{2} P_{z}^{2}\right)$ denotes nucleon mass and higher-twist contributions suppressed by powers of the large nucleon momentum. The flavor indices in $q, \tilde{q}$, and $C$ are implied. When $-1<y<0$, the distributions refer to the antiquark distributions.

Since the proposal of LaMET, remarkable progress has been made in both theoretical aspect and lattice calculations. It should be pointed out that these developments are achieved in an interactive way. The LaMET was first used to calculate the proton isovector quark distribution $f_{u-d}$ [15-20], including the unpolarized, polarized, and transversity cases, and subsequently to the meson distribution amplitudes [21,22]. The first lattice studies used the matching coefficients at one-loop order in a transversemomentum cutoff scheme [23-25]. However, as was found in Ref. [23], the original quasi-PDF suffers from an ultraviolet (UV) linear divergence which might pose a severe problem for the renormalization of its lattice matrix elements [26-28]. Then much attention has been paid to the renormalization property [29-37], and finally the multiplicative renormalizability of quasi-PDF in coordinate space in the continuum was proven to all orders in the strong coupling constant $\alpha_{s}$ [34,35]. This finding has further motivated the lattice analysis of nonperturbative renormalization (NPR) of the quasi-PDF [36,38,39] in the RI/ MOM scheme [40], and the calculation of the matching coefficients between the RI/MOM quasi-PDFs and $\overline{\mathrm{MS}}$ PDFs [11]. Besides the renormalization, the finite nucleon mass corrections were also worked out to all orders of $M^{2} / P_{z}^{2}$ [17], and higher-twist $\mathcal{O}\left(\Lambda_{\mathrm{QCD}}^{2} / x^{2} P_{z}^{2}\right)$ effects were numerically removed by extrapolating the results at several $P_{z}$ values to infinite momentum [15,17]. Based on these studies, calculations of the isovector quark PDF at physical pion mass have become available [20,41-43]. Potential operator mixing in the lattice renormalization of the quasi-PDF has also been investigated [33,36,38,39], and the mixing pattern classified in Ref. [44]. Ways to reduce the systematic uncertainties from Fourier transforming the spatial correlation at long distance were proposed in 
Refs. [41,45]. The LaMET was also attempted to study transverse-momentum-dependent distributions [46-53], as well as the gluon PDF [54-59].

In addition to LaMET, other interesting approaches have been proposed in recent years to calculate the PDFs from lattice QCD. For example, one can extract the PDFs from a class of "lattice cross sections" [13,14], while a smeared quasi-PDF in the gradient flow method was proposed to sweep the power divergence in the lattice calculation $[60,61]$. One can also study a pseudodistribution [62], which shows interesting renormalization features [12,63-65]. Moreover there are proposals using current-current correlators to compute the hadronic tensor [66,67], or the higher moments of the PDF, light-cone distribution amplitudes, etc. [67-72]. These different approaches are subject to their own systematics, but they can be compared to each other.

It was argued that the power divergent mixing between local moment operators may spoil the renormalization of quasi-PDFs [27,28], however, such a problem dissolves in LaMET since one first needs to take the continuum limit of the quasi-PDF after renormalization on the lattice, and then match it to obtain the $x$ dependence of the PDF. The factorization has been derived rigorously $[12,13]$ in the continuum, and one only needs to focus on the renormalization of the nonlocal spatial correlator only. Thus the renormalization of local moment operators is irrelevant to quasi-PDF. Besides, there are also confusions on the LaMET matching between Minkowskian and Euclidean matrix elements of the quasi-PDF [73], which have been clarified in Refs. [74,75].

Most of the available lattice calculations have used $\Gamma=\gamma^{z}$ (except $[42,43]$ ) for the unpolarized quasi-PDF, which is now known to mix with the scalar quasi-PDF operator $O_{I}$ at $O\left(a^{0}\right)[33,36,44]$. This operator mixing introduces an additional systematic uncertainty in nonperturbative renormalization [36,38,39,41], thus limiting the accuracy of the extracted PDF. On the contrary, the $\Gamma=\gamma^{t}$ case is free from operator mixing with $O_{I}$ at $O\left(a^{0}\right)$ $[33,36,44]$. Therefore, it is highly desirable to start from the quasi-PDF with $\Gamma=\gamma^{t}$. This is one main motif of this study.

In this work, we will carry out a lattice calculation of the unpolarized isovector quark distribution from the quasi-PDF with $\Gamma=\gamma^{t}$ with the same nonperturbative renormalization procedure as for the $\Gamma=\gamma^{z}$ case in Ref. [39]. The calculation is performed using clover fermions on a CLS ensemble of gauge configurations with $N_{f}=2+1$ (degenerate up/down, and strange) flavors under an open boundary condition [76] with pion mass $M_{\pi}=356 \mathrm{MeV}$ and lattice spacing $a=0.086 \mathrm{fm}$ [77]. We will examine the dependence on the nucleon momentum $P_{z}$ and the RI/MOM scales $p_{z}^{R}, \mu_{R}$, as well as on choices of the projection operator for the amputated Green's function in RI/MOM renormalization. Due to large uncertainties, it is hard to see the sea quark asymmetry observed in early studies which were performed without lattice renormalization [15-18]. In the future we plan to analyze CLS ensembles with a better accuracy and down to physical masses and $a<0.04 \mathrm{fm}$, both for $\left(m_{s}+m_{u}+m_{d}\right)$ fixed to its physical value and for physical $m_{s}$ using flavor $\mathrm{SU}(3)$ and $\mathrm{SU}(2)$ extrapolations.

The rest of this paper is organized as follows. In Sec. II, we briefly review the procedure of nonperturbative renormalization and matching of the quasi-PDF in the RI/MOM scheme, in particular the explicit one-loop matching coefficient for the $\Gamma=\gamma^{t}$ case. In Sec. III, we describe the details of lattice simulation of the hadronic matrix elements as well as its nonperturbative renormalization. Systematic errors in the calculation are also discussed in this section. In Sec. IV, we present our results on the $x$ dependence of the unpolarized isovector quark PDF with the statistical and systematic uncertainties, and the last section contains the summary of our work.

\section{NONPERTURBATIVE RENORMALIZATION AND MATCHING}

To recover the continuum limit of a quasi-PDF matrix element, nonperturbative renormalization on the lattice is required to deal with linear and logarithmic UV divergences. In this work, we follow the RI/MOM scheme elaborated in Refs. [11,39], and match the result to the $\overline{\mathrm{MS}}$ PDF with the one-loop matching coefficient [11].

\section{A. RI/MOM renormalization on the lattice}

The spatial correlator $O_{\Gamma}(z)$ has been proven to be multiplicatively renormalizable in coordinate space in the continuum [34,35], which enables the renormalization in RI/MOM scheme [40].

For each value of $z$, the RI/MOM renormalization factor $Z$ is obtained by requiring loop corrections for the matrix element of a quasi-PDF operator vanish in an off-shell quark state at a given momentum:

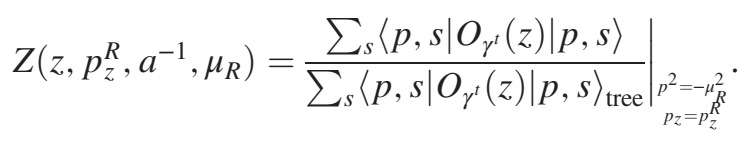

The bare matrix element $\sum_{s}\left\langle p, s\left|O_{\gamma^{t}}(z)\right| p, s\right\rangle$ will be calculated on the lattice from the amputated Green's function $\Lambda_{\gamma^{t}}(p, z)$ of $O_{\gamma^{t}}(z)$, with a projection operator $\mathcal{P}$ for the Dirac matrix:

$$
\sum_{s}\left\langle p, s\left|O_{\gamma^{t}}(z)\right| p, s\right\rangle=\operatorname{Tr}\left[\Lambda_{\gamma^{t}}(z, p) \mathcal{P}\right] .
$$

Due to the breaking of Lorentz covariance in $O_{\Gamma}(z)$, the RI/MOM subtraction depends on two scales $\mu_{R}$ and $p_{z}^{R}$. As a result, the renormalization factor $Z\left(z, p_{z}^{R}, a^{-1}, \mu_{R}\right)$ depends on the lattice spacing as well as on the two $\mathrm{RI} / \mathrm{MOM}$ scales $\mu_{R}$ and $p_{z}^{R}$. 
Based on the symmetry of $O_{\Gamma}(z)$ on the lattice, the amputated Green's function $\Lambda_{\gamma^{t}}(p, z)$ is not only proportional to the tree-level result $\gamma^{t}$, but also includes two other independent Lorentz structures:

$\Lambda_{\gamma^{t}}(p, z)=\tilde{F}_{t}(p, z) \gamma^{t}+\tilde{F}_{z}(p, z) \frac{p_{t} \gamma^{z}}{p_{z}}+\tilde{F}_{p}(p, z) \frac{p_{t} \not p}{p^{2}}$.

In the above $\tilde{F}_{i}$ 's are independent form factors that are invariant under the hypercubic group $H(4)$. According to Eq. (8) the RI/MOM renormalization factor $Z$ will also depend on the projection operator $\mathcal{P}$. One can choose to single out $\tilde{F}_{t}$ only [11], which we call the minimal projection. This projection has the simplest form but captures all the UV divergence in $\Lambda_{\gamma^{t}}(p, z)$. Optionally, one can choose $\mathcal{P}=\not p /\left(4 p^{t}\right)$, which we call the $\not p$ projection. The renormalization factors $Z$ with the minimal and $\not p$ projections are defined as

$$
\begin{aligned}
& \left.Z_{m p}\left(z, p_{z}^{R}, a^{-1}, \mu_{R}\right) \equiv \tilde{F}_{t}(p, z)\right|_{\substack{p^{2}=-\mu_{R}^{2} \\
p_{z}=p_{z}^{R}}}, \\
& Z_{p p}\left(z, p_{z}^{R}, a^{-1}, \mu_{R}\right) \\
& \left.\equiv\left[\tilde{F}_{t}(p, z)+\tilde{F}_{z}(p, z)+\tilde{F}_{p}(p, z)\right]\right|_{\substack{p^{2}=-\mu_{R}^{2} \\
p_{z}=p_{z}^{R}}} .
\end{aligned}
$$

The bare nucleon matrix element from a lattice calculation in coordinate space

$$
\tilde{h}\left(z, P_{z}, a^{-1}\right)=\frac{1}{2 P^{0}}\left\langle P\left|O_{\gamma^{t}}(z)\right| P\right\rangle
$$

is renormalized according to

$$
\begin{aligned}
& \tilde{h}_{R}\left(z, P_{z}, p_{z}^{R}, \mu_{R}\right) \\
& \quad=\left.Z^{-1}\left(z, p_{z}^{R}, a^{-1}, \mu_{R}\right) \tilde{h}\left(z, P_{z}, a^{-1}\right)\right|_{a \rightarrow 0} .
\end{aligned}
$$

Here $\tilde{h}_{R}\left(z, P_{z}, p_{z}^{R}, \mu_{R}\right)$ is the continuum limit of the renormalized matrix element. Consequently, the quasi-PDF
$\tilde{q}_{R}\left(x, P_{z}, p_{z}^{R}, \mu_{R}\right)$ in the RI/MOM scheme is obtained through the Fourier transformation of $\tilde{h}_{R}\left(z, P_{z}, p_{z}^{R}, \mu_{R}\right)$ :

$\tilde{q}_{R}\left(x, P_{z}, p_{z}^{R}, \mu_{R}\right)=P_{z} \int \frac{d z}{2 \pi} e^{i x P_{z} z} \tilde{h}_{R}\left(z, P_{z}, p_{z}^{R}, \mu_{R}\right)$.

In RI/MOM, $\tilde{h}_{R}\left(z, P_{z}, p_{z}^{R}, \mu_{R}\right)$ and $\tilde{q}_{R}\left(x, P_{z}, p_{z}^{R}, \mu_{R}\right)$ are independent of the UV regulator, and the one-step matching between the quasi-PDF and $\overline{\mathrm{MS}}$ PDF can be carried out in the continuum theory with dimensional regularization [11].

The quasi-PDFs will eventually be matched to the same $\overline{\mathrm{MS}} \mathrm{PDF}$, and the two projections with $Z_{m p}$ and $Z_{\not p}$ should generate the same result. However, the matching coefficient can only be calculated at a fixed loop order, hence remanent dependence on the projection operator is inevitable.

Using the same logic one reaches the conclusion that the quasi-PDF's dependence on the RI/MOM scales $\mu_{R}$ and $p_{z}^{R}$ should also be fully canceled by the matching coefficient. Any fixed-order matching calculation will inevitably lead to a residual $\mu_{R}, p_{z}^{R}$, and $P_{z}$ dependence of the final result for the PDF. These dependencies should be carefully studied and included in the systematic uncertainties.

\section{B. One-loop matching for quasi-PDF and PDF}

To obtain the matching coefficient between the quasi$\operatorname{PDF} \tilde{q}_{R}\left(x, P_{z}, p_{z}^{R}, \mu_{R}\right)$ and light-cone PDF $q(x, \mu)$, one can calculate the off-shell quark matrix elements in perturbation theory. In the following, the calculation will be conducted in Landau gauge for both minimal and $\not p$ projections. See Sec. 1 of the Appendix for the results in a general covariant gauge with a general Lorentz structure.

The lowest-order quark quasi-PDF is

$$
\tilde{q}^{(0)}(x)=\delta(1-x)
$$

At one-loop order, it is

$$
\tilde{q}^{(1)}(x, p, \rho)=\operatorname{Tr}\left[\left(\left[\tilde{f}_{t}(x, \rho)\right]_{+} \gamma^{t}+\left[\tilde{f}_{z}(x, \rho)\right]_{+} \frac{p_{t}}{p_{z}} \gamma^{z}+\left[\tilde{f}_{p}(x, \rho)\right]_{+} \frac{p_{t} \not p}{p^{2}}\right) \mathcal{P}\right] .
$$

The $\tilde{f}_{i} \mathrm{~s}$ are

$$
\tilde{f}_{t}(x, \rho)=\frac{\alpha_{s} C_{F}}{2 \pi} \begin{cases}\frac{8 x^{2}(1-x)-x \rho(13-10 x)+3 \rho^{2}}{2(1-x)(1-\rho)\left(4 x-4 x^{2}-\rho\right)}+\frac{4 x(2-x)-x \rho-3 \rho}{4(1-x)(1-\rho)^{3 / 2}} \ln \frac{2 x-1+\sqrt{1-\rho}}{2 x-1-\sqrt{1-\rho}} & x>1 \\ \frac{x(-7+4 x)+3 \rho}{2(1-x)(1-\rho)}+\frac{4 x(2-x)-\rho(3+x)}{4(1-x)(1-\rho)^{3 / 2}} \ln \frac{1+\sqrt{1-\rho}}{1-\sqrt{1-\rho}} & 0<x<1, \\ -\frac{8 x^{2}(1-x)-x \rho(13-10 x)+3 \rho^{2}}{2(1-x)(1-\rho)\left(4 x-4 x^{2}-\rho\right)}-\frac{4 x(2-x)-x \rho-3 \rho}{4(1-x)(1-\rho)^{3 / 2}} \ln \frac{2 x-1+\sqrt{1-\rho}}{2 x-1-\sqrt{1-\rho}} & x<0\end{cases}
$$




$$
\begin{aligned}
& \tilde{f}_{z}(x, \rho)=\frac{\alpha_{s} C_{F}}{2 \pi} \begin{cases}\frac{-32 x^{2}(1-x)^{2}(2 x-1)-4 x \rho\left(8-43 x+65 x^{2}-38 x^{3}+8 x^{4}\right)+\rho^{2}\left(5-41 x+42 x^{2}-8 x^{3}\right)+2 \rho^{3}(2-x)}{2(1-x)(1-\rho)^{2}\left(4 x-4 x^{2}-\rho\right)^{2}} & x>1 \\
+\frac{4-8 x+8 x^{2}+\rho\left(3-13 x+4 x^{2}\right)+2 \rho^{2}}{4(1-x)(1-\rho)^{5 / 2}} \ln \frac{2 x-1+\sqrt{1-\rho}}{2 x-1-\sqrt{1-\rho}} & 0<x<1 \\
\frac{-5+15 x-12 x^{2}-2 \rho(2-3 x)}{2(1-x)(1-\rho)^{2}}+\frac{4-8 x+8 x^{2}+\rho\left(3-13 x+4 x^{2}\right)+2 \rho^{2}}{4(1-x)(1-\rho)^{5 / 2}} \ln \frac{1+\sqrt{1-\rho}}{1-\sqrt{1-\rho}} & x<0 \\
-\frac{-32 x^{2}(1-x)^{2}(2 x-1)-4 x \rho\left(8-43 x+65 x^{2}-38 x^{3}+8 x^{4}\right)+\rho^{2}\left(5-41 x+42 x^{2}-8 x^{3}\right)+2 \rho^{3}(2-x)}{2(1-x)(1-\rho)^{2}\left(4 x-4 x^{2}-\rho\right)^{2}} & x<\end{cases} \\
& \tilde{f}_{p}(x, \rho)=\frac{\alpha_{S} C_{F}}{2 \pi} \begin{cases}\frac{16 x \rho(1-x)^{2}(1-6 x)-2 \rho^{2}\left(1-22 x+26 x^{2}-4 x^{3}\right)-\rho^{3}(7-6 x)}{2(1-\rho)^{2}\left(4 x-4 x^{2}-\rho\right)^{2}}+\frac{-\rho(8-12 x+\rho)}{4(1-\rho)^{5 / 2}} \ln \frac{2 x-1+\sqrt{1-\rho}}{2 x-1-\sqrt{1-\rho}} & x>1 \\
\frac{2-4 x+\rho(7-8 x)}{2(1-\rho)^{2}}+\frac{-\rho(8-12 x+\rho)}{4(1-\rho)^{5 / 2}} \ln \frac{1+\sqrt{1-\rho}}{1-\sqrt{1-\rho}} & 0<x<1, \\
-\frac{16 x \rho(1-x)^{2}(1-6 x)-2 \rho^{2}\left(1-22 x+26 x^{2}-4 x^{3}\right)-\rho^{3}(7-6 x)}{2(1-\rho)^{2}\left(4 x-4 x^{2}-\rho\right)^{2}}-\frac{-\rho(8-12 x+\rho)}{4(1-\rho)^{5 / 2}} \ln \frac{2 x-1+\sqrt{1-\rho}}{2 x-1-\sqrt{1-\rho}} & x<0\end{cases}
\end{aligned}
$$

and

$$
\rho=\frac{-p^{2}-i \epsilon}{p_{z}^{2}}
$$

with $i \epsilon$ giving the prescription to analytically extrapolate $\rho$ from $\rho<1$ (Minkowski) to $\rho>1$ (Euclidean). Notice that the vector current conservation guarantees that vertex corrections and wave function contributions can be combined into generalized plus functions [11]. These functions are defined with two arbitrary functions $h(x)$ and $g(x)$ :

$$
\int d x[h(x)]_{+} g(x)=\int d x h(x)[g(x)-g(1)] .
$$

For the light-cone PDF with the same off-shell IR regulation in Landau gauge, the tree-level contribution is

$$
q^{(0)}(x)=\delta(1-x)
$$

and the one-loop correction in the $\overline{\mathrm{MS}}$ scheme is

$$
\begin{aligned}
& q^{(1)}(x, p, \mu) \\
& \quad=\operatorname{Tr}\left[\left(\left[f_{+}\left(x, \frac{\mu^{2}}{p^{2}}\right)\right]_{+} \gamma^{+}+\left[f_{p}(x) \frac{p^{+} \not p}{p^{2}}\right]_{+}\right) \mathcal{P}\right] .
\end{aligned}
$$

Here

$$
\begin{aligned}
& f_{+}\left(x, \frac{\mu^{2}}{p^{2}}\right)= \frac{\alpha_{s} C_{F}}{2 \pi} \theta(x) \theta(1-x)\left[\frac{-5+10 x-6 x^{2}}{2(1-x)}\right. \\
&\left.+\frac{1+x^{2}}{1-x} \ln \frac{\mu^{2}}{-x(1-x) p^{2}}\right] \\
& f_{p}(x)=\frac{\alpha_{s} C_{F}}{2 \pi}(1-2 x) \theta(x) \theta(1-x) .
\end{aligned}
$$

To match the quasi-PDF to the light-cone PDF, one needs to take the on-shell limit $\left(p^{2} \rightarrow 0\right.$ or $\left.\rho \rightarrow 0\right)$ and the large-momentum limit $\left(p_{t} \rightarrow p_{z}\right)$ for the bare quasi-PDF

$$
\tilde{q}_{B}^{(1)}(x, \rho)=\tilde{q}^{(1)}\left(x,\left(p_{t} \rightarrow p_{z}, \vec{p}_{\perp}, p_{z}\right), \rho \rightarrow 0\right) .
$$

One can observe that both terms proportional to $\gamma^{t}$ and $\gamma^{z}$ in Eq. (14) approach light-cone operators in the large-momentum limit and the combination of them captures the correct collinear behavior. Therefore the bare quasi-PDF in minimal projection is defined to pick up the coefficient of $\gamma^{t}$ and $\gamma^{z}$ in Eq. (14):

$$
\left.\tilde{q}_{B}^{(1)}(x, \rho)\right|_{m p}=\left.\left[\tilde{f}_{t}(x, \rho)+\tilde{f}_{z}(x, \rho)\right]_{+}\right|_{\rho \rightarrow 0} .
$$

For the light-cone PDF, the coefficient of $\gamma^{+}$in Eq. (21) is used for minimal projection:

$$
\left.q^{(1)}(x, p, \mu)\right|_{m p}=f_{+}\left(x, \frac{\mu^{2}}{p^{2}}\right)_{+} .
$$

The bare matching coefficient is then derived as

$$
f_{1, m p}\left(x, \frac{p_{z}}{\mu}\right)_{+}=\left.\tilde{q}_{B}^{(1)}(x, \rho)\right|_{m p}-\left.q^{(1)}(x, p, \mu)\right|_{m p},
$$

where

$$
\begin{aligned}
& f_{1, m p}\left(x, \frac{p_{z}}{\mu}\right) \\
& =\frac{\alpha_{s} C_{F}}{2 \pi} \begin{cases}\frac{1+x^{2}}{1-x} \ln \frac{x}{x-1}+1 & x>1 \\
\frac{1+x^{2}}{1-x} \ln \frac{4 x(1-x) p_{z}^{2}}{\mu^{2}}-\frac{x(1+x)}{1-x} & 0<x<1 . \\
-\frac{1+x^{2}}{1-x} \ln \frac{x}{x-1}-1 & x<0\end{cases}
\end{aligned}
$$


In RI/MOM, the quasi-PDF is renormalized with an additional counterterm. We find that in the $|x| \rightarrow \infty$ limit, only $\tilde{f}_{t}(x, \rho)$ behaves as $1 /|x|$. When integrating over $x$, this term recovers UV divergence in the local limit $z=0$. Therefore, it is a natural choice to pick up the $\gamma^{t}$ term in Eq. (14) as a counterterm:

$$
\left.\tilde{q}_{C T}^{(1)}\left(x, r, \frac{p_{z}}{p_{z}^{R}}\right)\right|_{m p}=\left[\left|\frac{p_{z}}{p_{z}^{R}}\right| f_{2, m p}\left(1+\frac{p_{z}}{p_{z}^{R}}(x-1), r\right)\right]_{+} .
$$

Here $r=\mu_{R}^{2} /\left(p_{z}^{R}\right)^{2}$, and

$$
f_{2, m p}(x, r)=\tilde{f}_{t}(x, r)=\frac{\alpha_{s} C_{F}}{2 \pi} \begin{cases}\frac{-3 r^{2}+13 r x-8 x^{2}-10 r x^{2}+8 x^{3}}{2(r-1)(x-1)\left(r-4 x+4 x^{2}\right)}+\frac{-3 r+8 x-r x-4 x^{2}}{2(r-1)^{3 / 2}(x-1)} \tan ^{-1} \frac{\sqrt{r-1}}{2 x-1} & x>1 \\ \frac{-3 r+7 x-4 x^{2}}{2(r-1)(1-x)}+\frac{3 r-8 x+r x+4 x^{2}}{2(r-1)^{3 / 2}(1-x)} \tan ^{-1} \sqrt{r-1} & 0<x<1 . \\ -\frac{-3 r^{2}+13 r x-8 x^{2}-10 r x^{2}+8 x^{3}}{2(r-1)(x-1)\left(r-4 x+4 x^{2}\right)}-\frac{-3 r+8 x-r x-4 x^{2}}{2(r-1)^{3 / 2}(x-1)} \tan ^{-1} \frac{\sqrt{r-1}}{2 x-1} & x<0\end{cases}
$$

Finally, the matching coefficient $C$ in the factorization formula given in Eq. (5) is derived as

$$
\begin{aligned}
C\left(x, r, \frac{p_{z}}{\mu}, \frac{p_{z}}{p_{z}^{R}}\right) & =\delta(1-x)+\left.\left[\tilde{q}_{B}^{(1)}(x, \rho)-q^{(1)}(x, p, \mu)-\tilde{q}_{C T}^{(1)}\left(x, r, \frac{p_{z}}{p_{z}^{R}}\right)\right]\right|_{m p}+\mathcal{O}\left(\alpha_{s}^{2}\right) \\
& =\delta(1-x)+\left[f_{1, m p}\left(x, \frac{p_{z}}{\mu}\right)-\left|\frac{p_{z}}{p_{z}^{R}}\right| f_{2, m p}\left(1+\frac{p_{z}}{p_{z}^{R}}(x-1), r\right)\right]_{+}+\mathcal{O}\left(\alpha_{s}^{2}\right) .
\end{aligned}
$$

Here the coupling $\alpha_{s}(\mu)$ is in the standard $\overline{\mathrm{MS}}$ scheme. Note that the antiquark distribution is mapped into the region $-1<y<0$ by setting $q(y)=-\bar{q}(-y)$.

For $\not p$ projection, one has the bare quasi-PDF

$$
\left.\tilde{q}_{B}^{(1)}(x, \rho)\right|_{\not p}=\left[\tilde{f}_{t}(x, \rho)+\tilde{f}_{z}(x, \rho)+\tilde{f}_{p}(x, \rho)\right]_{+} .
$$

The light-cone PDF with a similar projection is

$$
\left.q^{(1)}(x, p, \mu)\right|_{\not p}=\left[f_{+}\left(x, \frac{\mu^{2}}{p^{2}}\right)+f_{p}(x)\right]_{+} .
$$

Under this projection, the matching coefficient for the bare quasi-PDF coincides with Eq. (27):

$$
\begin{aligned}
f_{1, \not}\left(x, \frac{p_{z}}{\mu}\right)_{+} & =\left.\tilde{q}_{B}^{(1)}(x, \rho)\right|_{\not p}-\left.q^{(1)}(x, p, \mu)\right|_{\not} \\
& =f_{1, m p}\left(x, \frac{p_{z}}{\mu}\right)_{+} .
\end{aligned}
$$

The counterterm can be obtained by calculating with $\mathcal{P}=\not p /\left(4 p^{t}\right)$ :

$$
f_{2, \not p}(x, r)=\frac{\alpha_{s} C_{F}}{2 \pi} \begin{cases}\frac{3-3 r-2 x}{2(r-1)(x-1)}+\frac{4 r x-8 x^{2}+8 x^{3}}{\left(r-4 x+4 x^{2}\right)^{2}}+\frac{2-2 r-r x+2 x^{2}}{(r-1)^{3 / 2}(x-1)} \tan ^{-1} \frac{\sqrt{r-1}}{2 x-1} & x>1 \\ \frac{3-3 r-2 x+4 x^{2}}{2(r-1)(1-x)}+\frac{-2+2 r+r x-2 x^{2}}{(r-1)^{3 / 2}(1-x)} \tan ^{-1} \sqrt{r-1} & 0<x<1 . \\ -\frac{3-3 r-2 x}{2(r-1)(x-1)}-\frac{4 r x-8 x^{2}+8 x^{3}}{\left(r-4 x+4 x^{2}\right)^{2}}-\frac{2-2 r-r x+2 x^{2}}{(r-1)^{3 / 2}(x-1)} \tan ^{-1} \frac{\sqrt{r-1}}{2 x-1} & x<0\end{cases}
$$

The corresponding RI/MOM matching coefficient is obtained by replacing $m p$ with $\not p$ in Eq. (31), and the difference between $f_{2, \gamma}$ and $f_{2, m p}$ vanishes in the $p_{z}^{R}=0$ limit. The matching coefficient with $\Gamma=\gamma^{z}$ is also given in Sec. 2 of the Appendix.

\section{LATTICE CALCULATION OF PDF}

\section{A. Lattice matrix elements}

In this section, we give the results of a lattice-QCD calculation using clover valence fermions on the CLS
$32^{3} \times 962+1$ flavor clover fermion ensemble H102 with lattice spacing $a=0.086 \mathrm{fm}$, pion mass $M_{\pi}=356 \mathrm{MeV}$, and box size $L \approx 2.7 \mathrm{fm}\left(M_{\pi} L \approx 4.9\right)$ [77]. We use $\kappa_{l}=$ 0.136865 and $C_{S W}=1.98625$ for the valence clover fermion. We apply APE smearing [78] with size $=2.5 a$ twice in the source/sink smearing and also in the quasi-PDF operator $O_{\Gamma}$, but not in the fermion propagators.

First of all, we will explore the nonperturbative renormalization in the RI/MOM scheme. Following Ref. [39], we use Landau gauge fixed wall sources (while limiting the source in the time slice range $t \in[32,64]$ to avoid the 
boundary effect from the open boundary condition at $t=0$ ), and generate the propagators with the momentum modes listed in Table I. The four digits in brackets correspond to three spatial components and the energy component in units of $2 \pi / L$. The $z$ direction can be selected by setting the Wilson link along any of the three spatial directions. Thus these results approximately cover three values of $\mu_{R}=\sqrt{-\left(p^{R}\right)^{2}}(2.4,3.2$, and $3.9 \mathrm{GeV}$, corresponding to $a^{2} \mu_{R}^{2}=1.1,2.0$, and 2.8), and $p_{z}^{R}=$ $\{0,1,2, \ldots\} ,2 \pi / L$ up to the upper limit $p_{z}^{R}<\mu_{R}$. Note that in deriving these momentum modes we have required the spatial components of a given momentum mode to differ from each other, and adjusted $p_{t}^{R}$ to ensure $\mu_{R}$ invariant (within 2\%). These choices allow us to explore the dependence on each component of $p$, but one should be cautious that the results may suffer from sizable discretization errors since the normal constraint $\frac{\sum_{\mu} a^{4} p_{\mu}^{4}}{\left(\sum_{\mu} a^{2} p_{\mu}^{2}\right)^{2}}<0.3$ is not respected. These discretization errors will be investigated in the future.

As shown in Eq. (31), the one-loop matching formula primarily depends on the combination $r=\mu_{R}^{2} /\left(p_{z}^{R}\right)^{2}$ but is independent of $p_{t}^{R}$. In Fig. 1, the $Z_{m p}$ and $Z_{\not p h}-Z_{m p}$ at fixed $z \sim 0.5 \mathrm{fm}$ are plotted as a function of $p_{z}^{R} / \mu_{R}=1 / \sqrt{r}$. From this figure, one can see that the NPR factors, both real and imaginary parts, only show the dependence on $r$ regardless of the values of $p_{z}^{R}$ or $\mu_{R}$, with $p_{z}^{R} / \mu_{R}<0.4$.

In Fig. 2, we show $1 / Z_{m p}$ and $1 / Z_{\not p}-1 / Z_{m p}$ as functions of the Wilson link length $z$, with the same $\mu_{R}=$ $3.2 \mathrm{GeV}$ and $p_{z}^{R}=1.4 \mathrm{GeV}$ and two different values of $p_{t}^{R}=0.9$ and $2.7 \mathrm{GeV}$. As shown in this figure, the $1 / Z_{m p}$ and $1 / Z_{\not p}-1 / Z_{m p}$ with the two different $p_{t}^{R}$ 's are close to each other for all $z$ (the curves with the same color). This is consistent with the one-loop matching formula. At $z<0.3 \mathrm{fm}$, the real part of $1 / Z_{\not p}-1 / Z_{m p}$ with the two different $p_{t}^{R}$, s can be slightly nonzero, but it is still smaller than $1 / Z_{\not p}$ by 2 orders of magnitude.

In the following we will take $p_{z}^{R}$ to be zero and estimate the systematic uncertainty from the $p_{z}^{R}$ dependence by varying the $p_{z}^{R}$.

TABLE I. Momentum modes used in the NPR analysis. The four digits (in units of $2 \pi / L$ ) in brackets correspond to three spatial momentum components and the energy component. The $z$ direction can be selected by setting the Wilson link along any of the three spatial directions. Thus these momentum modes approximately cover three choices of $\mu_{R}=\sqrt{-\left(p^{R}\right)^{2}}$ and several sets of $p_{z}^{R}=2 \pi i / L(i=0,1,2, \ldots)$.

\begin{tabular}{lc}
\hline$a^{2} \mu_{R}^{2}$ & Momentum modes $p^{R}$ \\
\hline$[1.109,1.118]$ & $(5,2,0,0)(4,3,1,5)$ \\
{$[1.957,1.966]$} & $(5,5,0,3)(6,3,2,4)(5,4,1,9)$ \\
{$[2.814,2.832]$} & $(6,5,1,10)(7,4,2,6)(6,3,0,16)$ \\
\hline
\end{tabular}
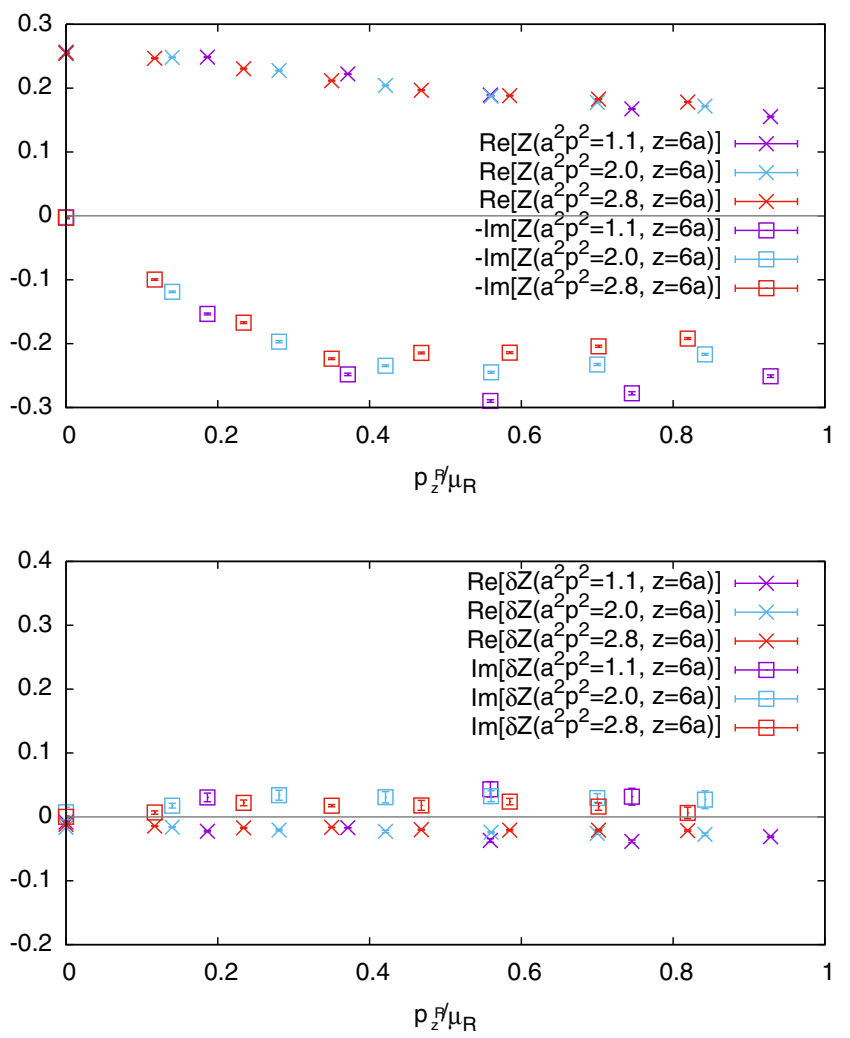

FIG. 1. (Top panel) The NPR $Z=Z_{m p}$ and (bottom panel) $\delta Z=Z_{\not p}-Z_{m p} \quad$ at $\quad z=6 a \quad(\approx 0.5 \mathrm{fm})$ as a function of $p_{z}^{R} / \mu_{R}=1 / \sqrt{r}$, with various $\mu_{R}$. At $p_{z}^{R}=0, Z$ is real and $\delta Z / Z$ is less than $5 \%$.

In the calculation of the nucleon matrix element, we use Gaussian momentum smearing [79] for the quark field

$$
\begin{aligned}
\psi(x) & \rightarrow S_{\mathrm{mom}} \psi(x) \\
& =\frac{1}{1+6 \alpha}\left[\psi(x)+\alpha \sum_{j} U_{j}^{\mathrm{APE}}(x) e^{i k \hat{e}_{j}} \psi\left(x+\hat{e}_{j}\right)\right],
\end{aligned}
$$

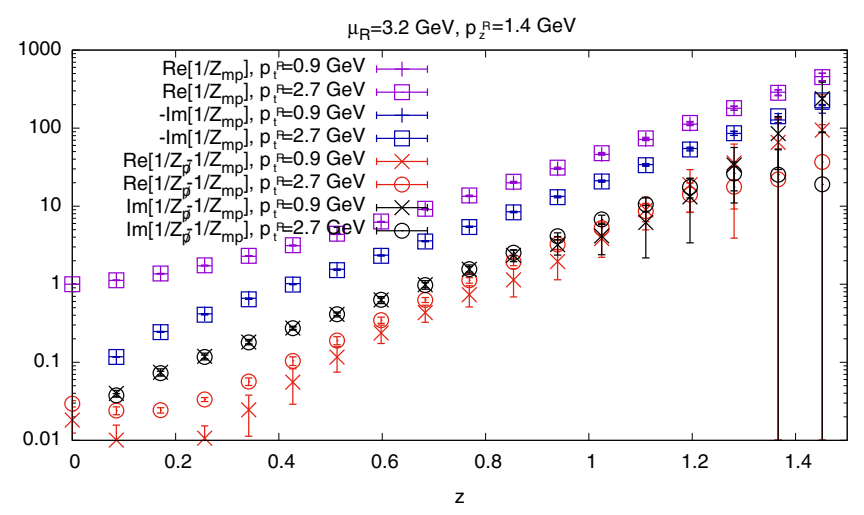

FIG. 2. The inverse of the minimum projection renormalization factor $1 / Z_{m p}$ and the difference $1 / Z_{\not p}-1 / Z_{m p}$, as a function of $z$ with the same $p_{z}^{R}$ and $\mu_{R}$, but different $p_{t}^{R}$. The crosses correspond to $p_{t}^{R}=0.9 \mathrm{GeV}$ and the open boxes (circles) are for $p_{t}^{R}=2.7 \mathrm{GeV}$. Most results show mild dependence on $p_{t}^{R}$. 
where $k$ is the desired momentum, $U_{j}^{\mathrm{APE}}(x)$ are the APE smeared gauge links in the $j$ direction, and $\alpha$ is a tunable parameter as in traditional Gaussian smearing.

Such a momentum source is designed to increase the overlap with nucleons of the desired boost momentum and we are able to reach higher-boosted momentum for the nucleon states than in the previous work [39]. Although in the exploratory study we varied the Gaussian smearing radius to better overlap with the largest momentum used in the calculation, the field smearing is still centered around zero momentum in momentum space. When we switch to the momentum smearing, the smearing center will be shifted to momentum $O(k)$, which will immediately allow us to reach higher boost momenta with better signal-tonoise ratios in the matrix elements. In this work, we use two values of nucleon boost momenta, $P_{z}=n \frac{2 \pi}{L}$, with $n \in\{4,5\}$, which corresponds to 1.8 and $2.3 \mathrm{GeV}$.
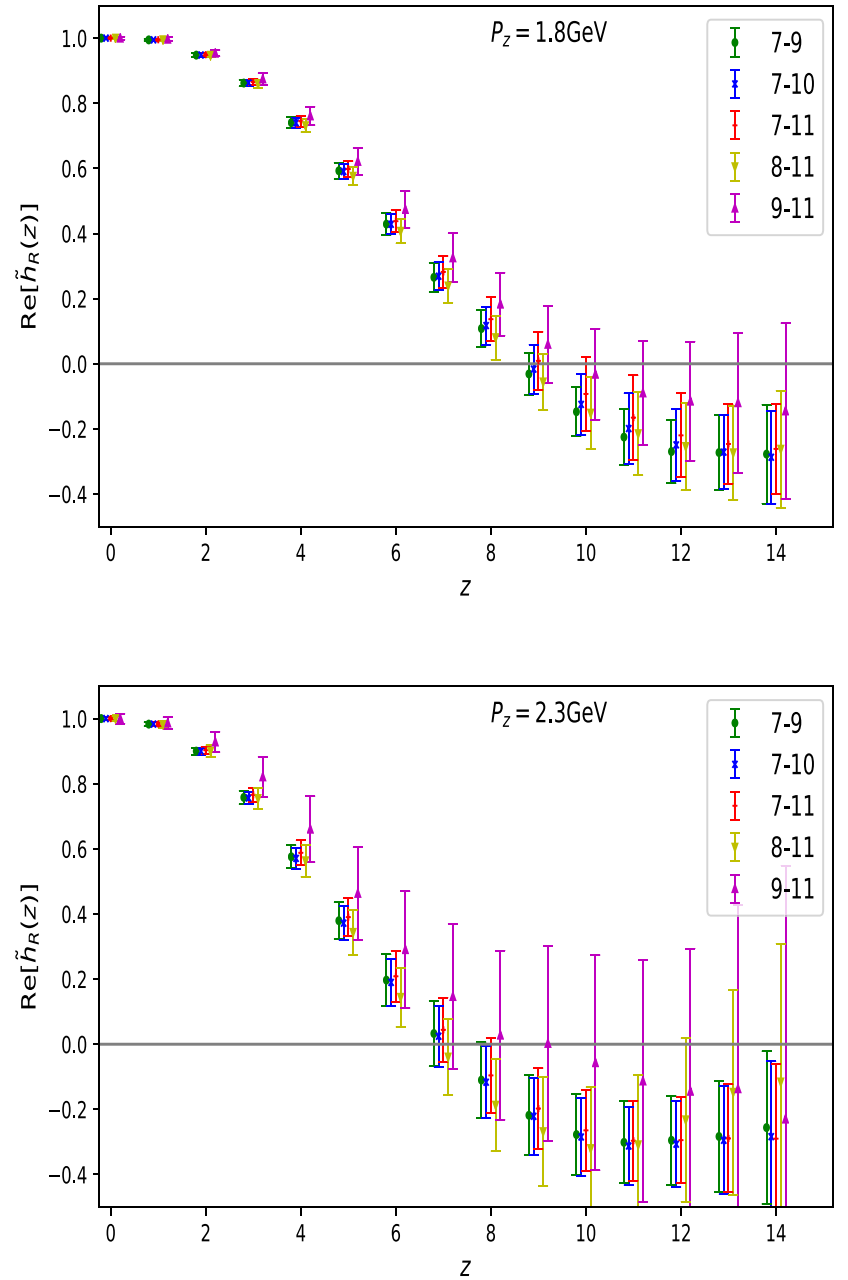

On the lattice, we calculate the time-independent and nonlocal in the $z$ direction correlators of a nucleon with finite- $P_{z}$ boost

$$
\tilde{h}_{\text {lat }}\left(z, P_{z}, \Gamma ; a^{-1}\right)=\left\langle 0 ; \vec{P}\left|O_{\Gamma}(z)\right| 0 ; \vec{P}\right\rangle \text {. }
$$

Here the state $|0 ; \vec{P}\rangle$ represents the ground (nucleon) state with momentum $\vec{P}=\left\{0,0, P_{z}\right\} . \Gamma=\gamma^{t}$ is used for the unpolarized parton distribution.

As the nucleon boost momentum increases, one anticipates that excited-state contributions are more severe; therefore, a careful study of the excited-state contamination is necessary. To do so, we calculate the nucleon matrix element $\tilde{h}_{\text {lat }}$ at five source-sink separations $t_{\text {seq }} \in$ $\{7,8,9,10,11\} \times 0.086 \mathrm{fm}$, with $\{4,4,8,8,16\}$ measurements on each of 2005 gauge configurations respectively in
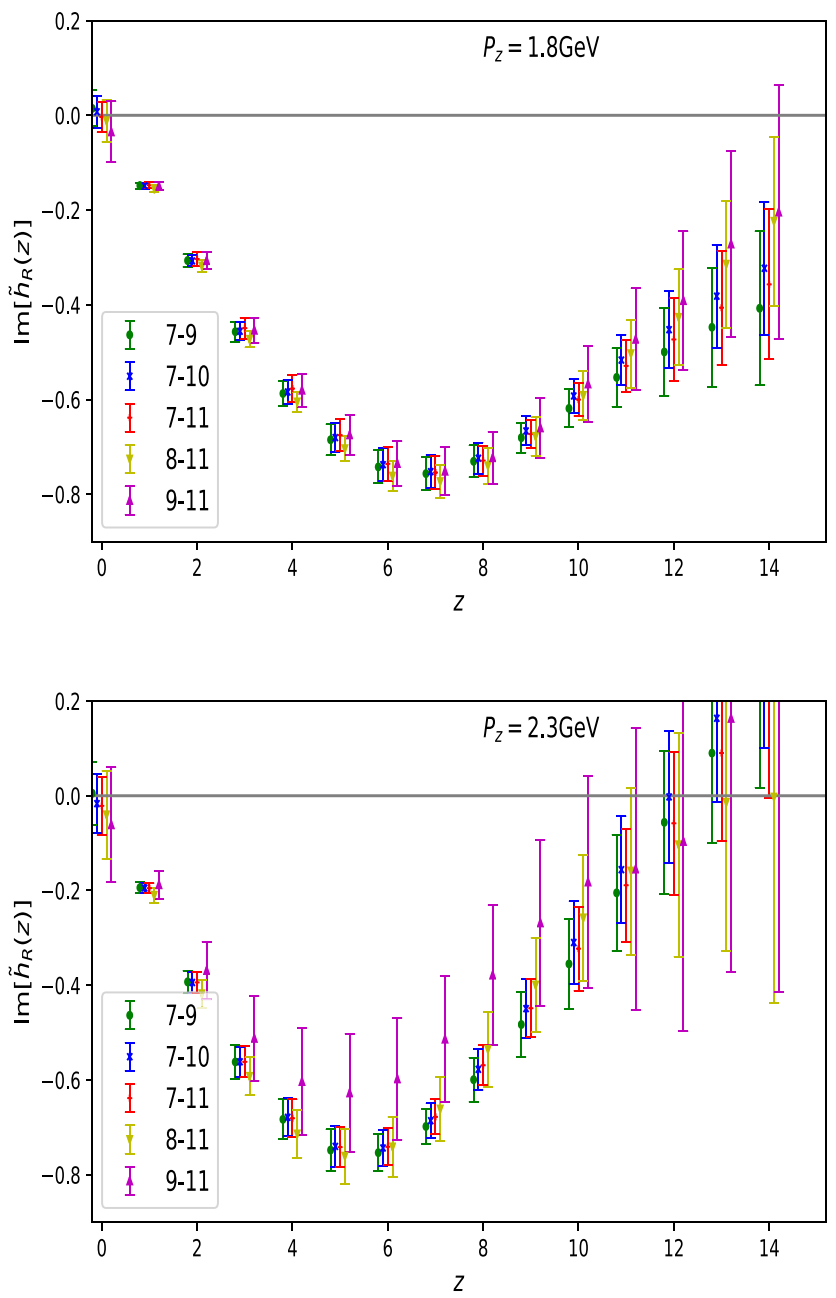

FIG. 3. The real (left panels) and imaginary (right panels) parts of the isovector nucleon matrix elements for unpolarized PDFs as functions of $z$ at different momenta, with $P_{z}=\frac{8 \pi}{L}=1.8 \mathrm{GeV}$ (top panels) and $\frac{10 \pi}{L}=2.3 \mathrm{GeV}$ (bottom panels) respectively. The $\mathrm{RI} / \mathrm{MOM}$ renormalization factors with $\left\{\mu, p_{z}^{R}\right\}=\{3.2,0\} \mathrm{GeV}$ and the normalization at $z=0$ are applied on the bare matrix elements to improve the visibility at large $z$. At a given positive $z$ value, the data are slightly offset to show the ground-state matrix element from the fits using different ranges; from left to right they are: $t_{\mathrm{seq}} \in[7,9],[7,10],[7,11],[8,11]$, and [9,11]. Different analyses are consistent within statistical errors, while the fits with separation 7 and 8 have smaller uncertainties compared to other cases. 
the $P_{z}=1.8 \mathrm{GeV}$ case, and of 2000 configurations in the $P_{z}=2.3 \mathrm{GeV}$ case. We use a multigrid algorithm [80,81] with the CHROMA software package [82] to speed up the inversion of the quark propagator. Following Ref. [83], each three-point (3pt) correlator $C_{\Gamma}^{(3 \mathrm{pt})}\left(t, t_{\mathrm{seq}}\right)$ can be decomposed as (assuming the source is at $t=0$ )

$$
\begin{aligned}
C^{3 \mathrm{pt}}\left(t, t_{\mathrm{seq}} ; P_{z}, \Gamma\right)= & \mathcal{A}_{0}^{2}\left\langle 0\left|O_{\Gamma}\right| 0\right\rangle e^{-E_{0} t_{\mathrm{seq}}} \\
& +\mathcal{A}_{1}^{2}\left\langle 1\left|O_{\Gamma}\right| 1\right\rangle e^{-E_{1} t_{\mathrm{seq}}} \\
& +\mathcal{A}_{1} \mathcal{A}_{0}\left\langle 1\left|O_{\Gamma}\right| 0\right\rangle e^{-E_{1}\left(t_{\mathrm{seq}}-t\right)} e^{-E_{0} t} \\
& +\mathcal{A}_{0} \mathcal{A}_{1}\left\langle 0\left|O_{\Gamma}\right| 1\right\rangle e^{-E_{0}\left(t_{\mathrm{seq}}-t\right)} e^{-E_{1} t}+\cdots,
\end{aligned}
$$

where $|n\rangle$ with $n>0$ represents the excited states. The operator is inserted at time $t$, and the nucleon state is annihilated at the sink time $t_{\text {seq }}$ (which is also the sourcesink separation). The spectrum weights $\mathcal{A}_{0,1}$ and energies
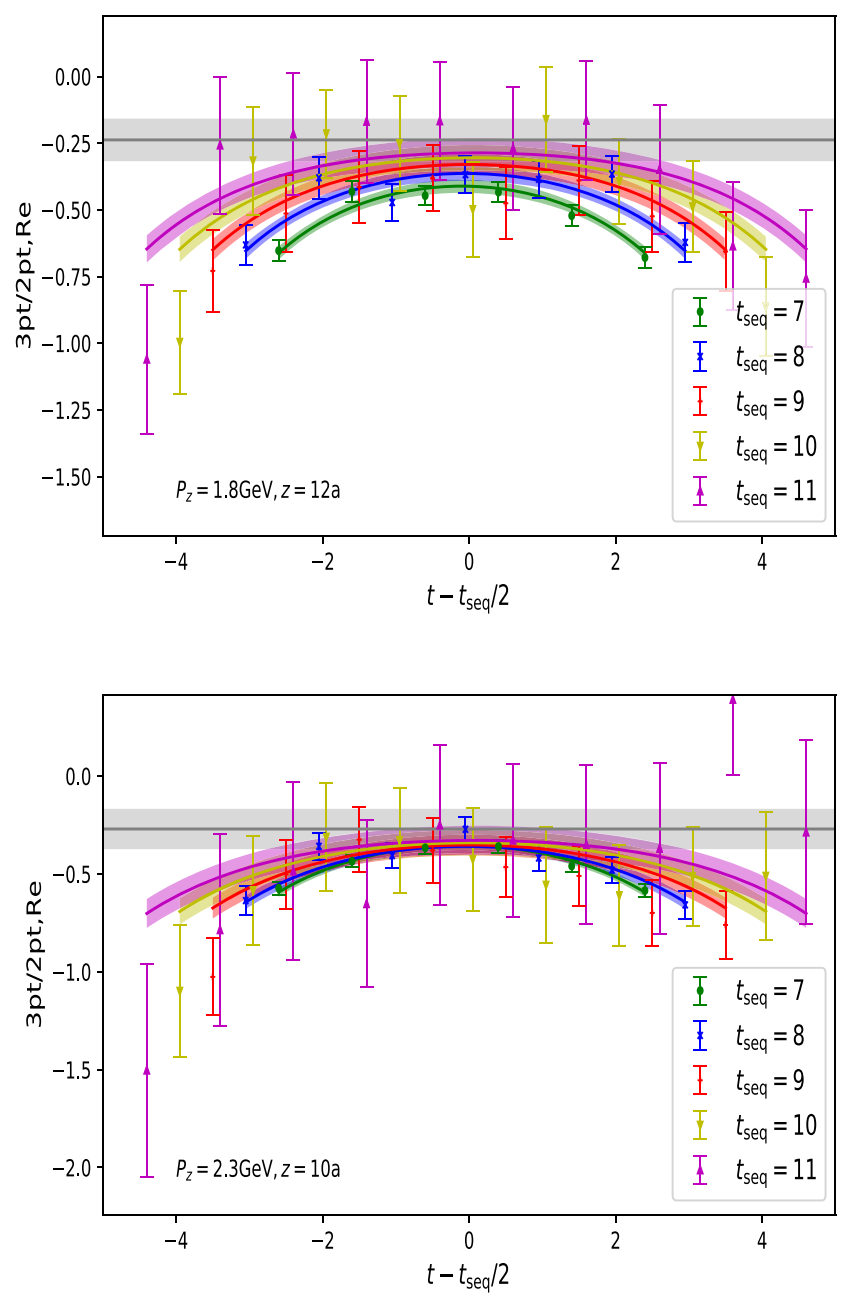

$E_{0,1}$ in Eq. (39) can be obtained from the two-point (2pt) correlator:

$$
C^{2 \mathrm{pt}}\left(t_{\mathrm{seq}} ; P_{z}\right)=\mathcal{A}_{0}^{2} e^{-E_{0} t_{\mathrm{seq}}}+\mathcal{A}_{1}^{2} e^{-E_{1} t_{\mathrm{seq}}}+\cdots .
$$

Eventually we apply the joint fit with the $3 p t$ functions at several $t_{\text {seq }}$ and $2 \mathrm{pt}$ function using the following form [83]:

$$
\begin{aligned}
\frac{C^{3 \mathrm{pt}}\left(t, t_{\mathrm{seq}}\right)}{C^{2 \mathrm{pt}}\left(t_{\mathrm{seq}}\right)} & =\frac{\tilde{h}_{\mathrm{lat}}+C_{2}\left(e^{-\Delta E t}+e^{-\Delta E\left(t_{\mathrm{seq}}-t\right)}\right)+C_{3} e^{-\Delta E t_{\mathrm{seq}}}}{1+C_{1} e^{-\Delta E t_{\mathrm{seq}}}}, \\
C^{2 \mathrm{pt}}(t) & =C_{0} e^{-E_{0} t}\left(1+C_{1} e^{-\Delta E t}\right),
\end{aligned}
$$

with $\Delta E=E_{1}-E_{0} . C_{0,1,2,3}$ and $E_{0,1}$ are free parameters. We limit the range of $t$ as $t \in\left[1, t_{\mathrm{seq}}-1\right]$ for the $3 \mathrm{pt} / 2 \mathrm{pt}$ ratio and $t \in[7,11]$ for the $2 \mathrm{pt}$ to make the $\chi^{2} /$ d.o.f. of the fit to be $\mathcal{O}(1)$. Using the ratio of $3 \mathrm{pt} / 2 \mathrm{pt}$ instead of the $3 \mathrm{pt}$ function itself can improve the stability of the fit, especially when $C^{2 \mathrm{pt}}(t)$ with $t<7$ is included in the fit.
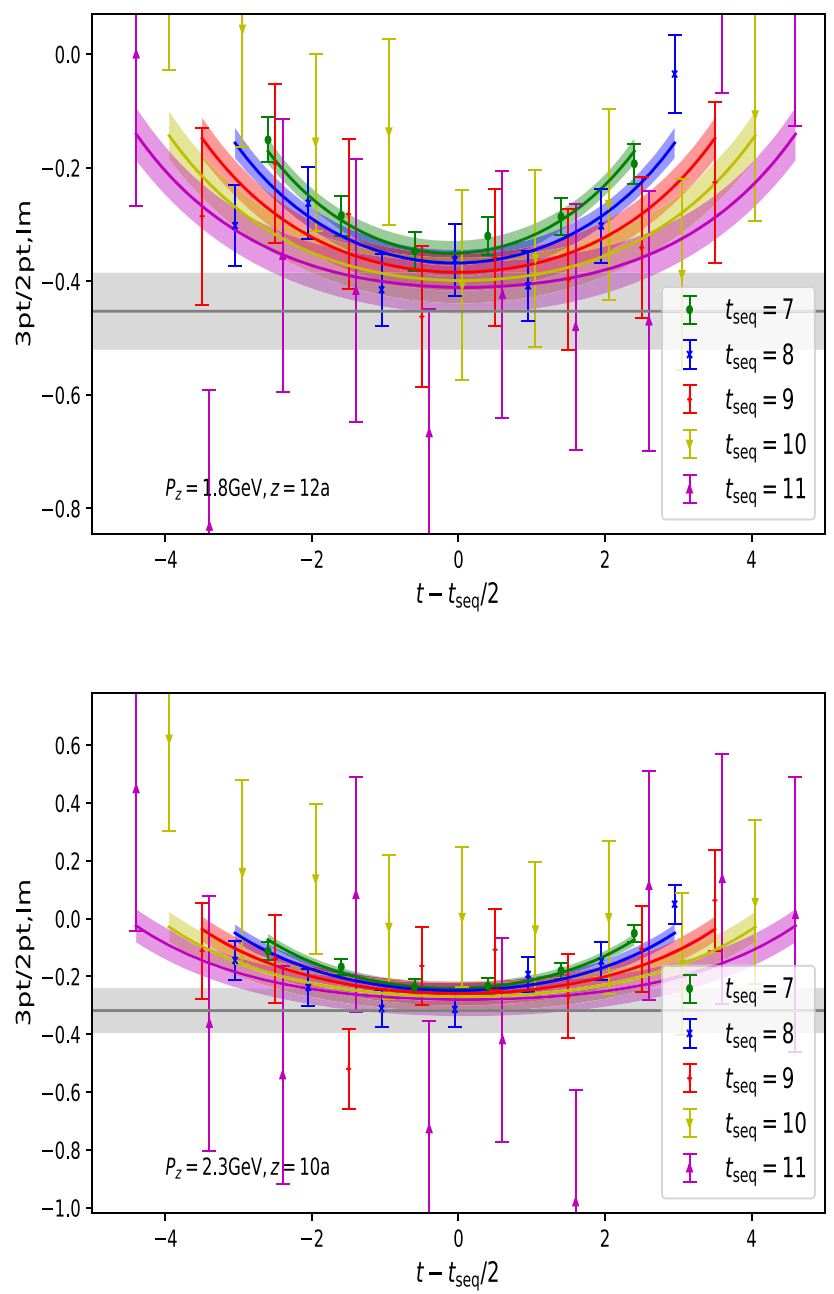

FIG. 4. The real (left panels) and imaginary (right panels) parts of the renormalized isovector nucleon matrix elements for unpolarized PDFs with $P_{z}, z=8 \pi / L, 12$ (top panels) and $10 \pi / L, 10$ (bottom panels), which correspond to $z P_{z} \sim 9.5$. The data points and the band predicted by the fit using $t_{\text {seq }} \in[7,11]$ agree with each other well. 
In Fig. 3, we show the ground-state nucleon matrix elements $\tilde{h}_{\text {lat }}\left(z, P_{z}, \gamma_{t}\right)$ obtained from five fits: using the separations $t_{\text {seq }} \in[7,9],[7,10],[7,11],[8,11]$, and $[9,11]$. (The data points correspond to the same $z$ but are shifted horizontally to enhance the visibility.) The data are further normalized by multiplying the renormalization factor with $\left\{\mu_{R}, p_{z}^{R}\right\}=\{3.2,0\} \mathrm{GeV}$ and the real part normalized to 1 at $z=0$. From this figure, one can see that there is no clear signal for excited-state contributions in any of these analyses. If the data with smallest two separations are dropped, uncertainties are getting much larger. In the fit, we keep the $C_{3}$ term to make a moderate estimate of the uncertainty even when this term is not statistically significant.

For a comparison between data and the fit, we show our results at large $z$ like $\left(P_{z}, z\right)=(8 \pi / L, 12 a)$ and $(10 \pi / L, 10 a)$ with $t_{\text {seq }} \in[7,11]$ in Fig. 4 . In these spatial separations, the real part of the matrix element seems to be negative. The ground-state contribution obtained from the fit is shown as the black band. As one can see, most data can be well described in the fit and thereby we use the two-state fits and the interval $t_{\mathrm{seq}} \in[7,11]$ to obtain the results in the rest of this paper.

The renormalized quasi-PDF matrix elements with two values of $P_{z}$ are plotted in Fig. 5, as a function of $z P_{z}$ for
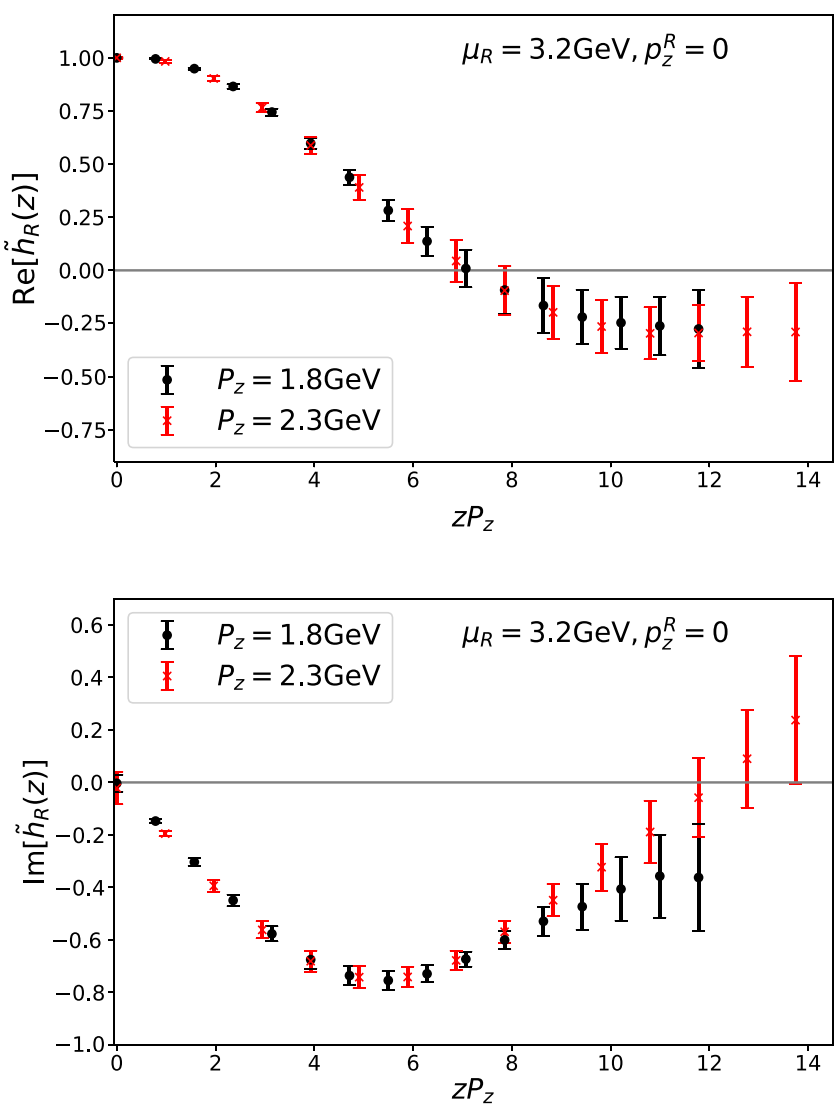

FIG. 5. The renormalized quasi-PDF matrix elements with $P_{z}=$ 1.8 and $2.3 \mathrm{GeV}$, using the minimal projection with $p_{z}^{R}=0$ and $\mu_{R}=3.2 \mathrm{GeV}$, as function of $z P_{z}$.
$p_{z}^{R}=0$ and $\mu_{R}=3.2 \mathrm{GeV}$. The results with different $P_{z}$ are consistent with each other within statistical uncertainties. This indicates that power corrections due to highertwist effects might not be sizable.

\section{B. Systematic uncertainties}

In this section, we will consider four systematic uncertainties, from Fourier transformation (FT), unphysical scales $p_{z}^{R}$ and $\mu_{R}$, projection used in the RI/MOM scheme, and inversion of the matching coefficient.

In the following, we explain the details to include these systematic uncertainties.

(1) Fourier transformation. As shown in Fig. 5, the $\tilde{h}_{R}(z)$ with $P_{z}=2.3 \mathrm{GeV}$ is consistent with zero when $z>12 a$. Thus in the standard matching from quasi-PDF to PDF, it is reasonable to truncate the results at $z=12 a$. With this spirit, the quasi-PDF and matched PDF using the standard FT are shown in the upper panel of Fig. 6, from which one can see the matched PDF shows an oscillatory behavior. A "derivative" method was proposed in Ref. [41] to cure this oscillatory behavior. To be concrete, one takes the derivative of the renormalized nucleon matrix elements $\partial_{z} \tilde{h}_{R}(z)$, whose Fourier transform differs from the original matrix element in a known way:

$$
\tilde{q}_{R}(x)=\int_{-\infty}^{\infty} \frac{d z}{2 \pi} \frac{i e^{i x P_{z} z}}{x} \partial_{z} \tilde{h}_{R}(z)
$$

From the above equation, one can see that when $\tilde{h}_{R}(z)$ goes to zero as $|z| \rightarrow \infty$, performing an integration by part and neglecting the boundary term will lead to the ordinary Fourier transformation. In implementing the derivative method in Eq. (41), one can perform the integration over $z$ in a discretized way, or interpolate the $\tilde{h}_{R}$ first. We have checked that the two methods give consistent results. With the same truncation as in the ordinary Fourier transformation, the result is shown in the lower panel of Fig. 6 and apparently the oscillatory behavior is less severe. Besides, results obtained using the derivative method are consistent with the standard FT method in most of the kinematics region except at small $x$. This is anticipated as the two methods only differ in the large- $z$ region where we have made the truncation. We show the difference as the dotdashed-blue line in Fig. 7, together with the error from varying the truncation from $z=10 a$ to $14 a$ (dottedgreen line).

Extending the lattice calculations restricted to a finite number of determinations of matrix elements to recapture all information on the PDF is a sophisticated problem. Thus it is necessary to point out that the derivative method we adopted in Eq. (41) can be viewed as a model-dependent treatment and might introduce some discretization uncertainties. This can possibly be overcome by using reconstruction methods; see Ref. [84] for detailed discussions. 
(2) Unphysical scales $p_{z}^{R}$ and $\mu_{R}$. There are two unphysical scales $p_{z}^{R}$ and $\mu_{R}$ introduced in RI/MOM. In principle, when matching the quasi-PDF matrix element onto a lightcone PDF, the dependence on these two scales in the matrix element should exactly cancel with that in the matching kernel. However, since the quasi-PDF matrix element is nonperturbatively renormalized on the lattice, while the matching coefficient is calculated at one-loop order in perturbation theory, there will be residual dependence on these two scales after the perturbative matching. To estimate the residual $p_{z}^{R}$ and $\mu_{R}$ dependence, we choose $p_{z}^{R}=0 \mathrm{GeV}$ and $\mu_{R}=3.2 \mathrm{GeV}$ as the central value, and vary $p_{z}^{R}$ from -1.4 to $1.4 \mathrm{GeV}$ (dashed-red line) and $\mu_{R}$ from 2.4 to $3.9 \mathrm{GeV}$ (thick-solid-orange line). The difference between these matched PDFs is treated as the systematics of the residual dependence on unphysical scales, in Fig. 7. As shown in the figure, the systematic uncertainty due to the $\mu_{R}$ dependence is small compared to the other sources, but the residual $p_{z}^{R}$ dependence could be sizable.
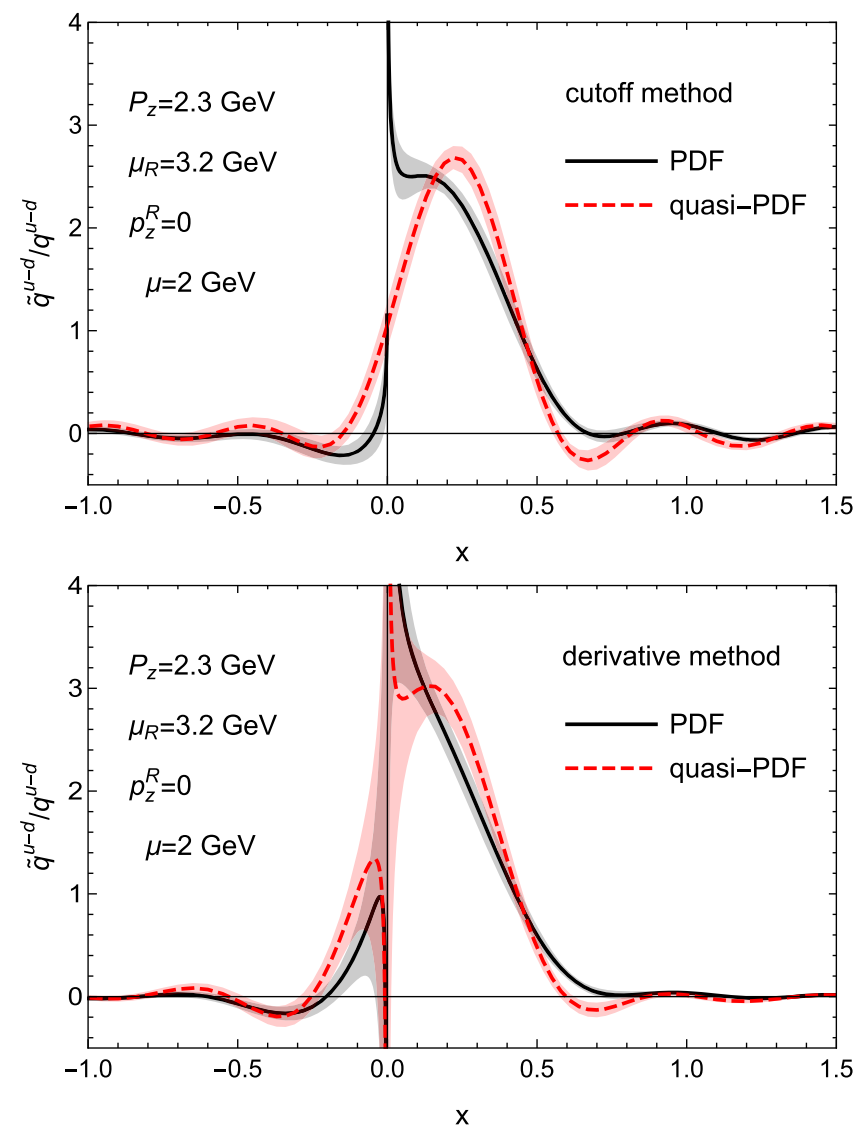

FIG. 6. The quasi-PDF (dashed-red lines) with nucleon boost momentum $2.3 \mathrm{GeV}$ and matched PDF (solid-black lines) at $\mu=$ $2 \mathrm{GeV}$ using minimal projection, with the RI/MOM parameters $p_{z}^{R}=0$ and $\mu_{R}=3.2 \mathrm{GeV}$. The upper and lower figures are obtained using the derivative and cutoff methods to perform Fourier transformation. The matching strategy has an important impact on the final results for PDFs.
(3) Dependencies on the projection. There are two projections discussed in this work: the minimum and " $p$ " projections. With $p_{z}^{R}=0$, the projection dependence in both the NPR factor are less than $5 \%$ for all the $z$, and vanishes in the one-loop perturbative matching. Thus one can expect the difference due to the projections to also be small, as depicted in the long-dashed-magenta lines in Fig. 7.

(4) Inversion of matching. To extract the PDF from the quasi-PDF, one needs to invert the factorization formula Eq. (5). This could be done by changing the sign of $\alpha_{s}$ in $C$, and convoluting the new matching coefficient with $\tilde{q}$. More explicitly, we have

$$
\begin{aligned}
q(x, \mu)= & \int_{-\infty}^{\infty} \frac{d y}{|y|} C^{\prime}\left(\frac{x}{y}, r, \frac{y P_{z}}{\mu}, \frac{y P_{z}}{p_{z}^{R}}\right) \tilde{q}\left(y, P_{z}, p_{z}^{R}, \mu_{R}\right) \\
& +\mathcal{O}\left(\frac{M^{2}}{P_{z}^{2}}, \frac{\Lambda_{\mathrm{QCD}}^{2}}{x^{2} P_{z}^{2}}, \alpha_{s}^{2}\right),
\end{aligned}
$$

where $C^{\prime}=C\left(\alpha_{s} \rightarrow-\alpha_{s}\right)$. We estimate the error due to inverting the factorization formula by starting from the PDF from a global analysis [85], applying Eq. (5) and then Eq. (42) to return to the PDF. This manipulation should give the same light-cone PDF. However, since the matching is only accurate up to $O\left(\alpha_{s}\right)$, the two results would differ, and the difference gives a good estimate of
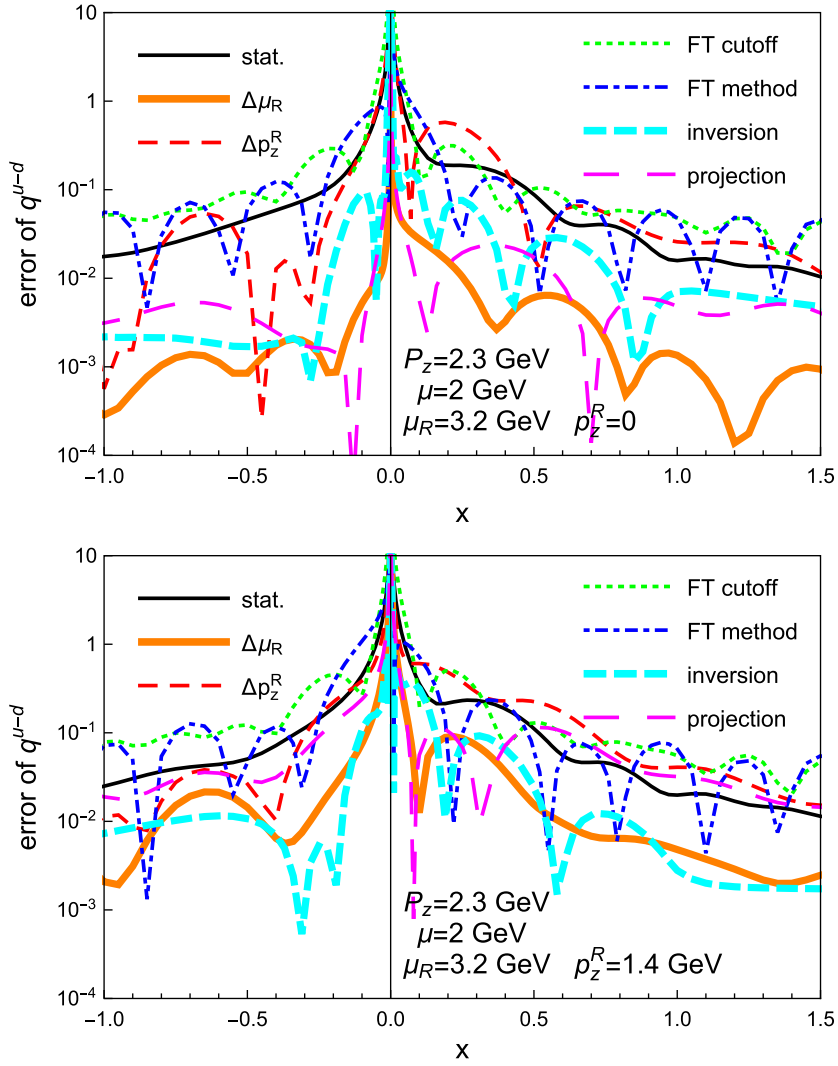

FIG. 7. Different contributions to the systematic errors. See the text for detailed information. 
the systematic error coming from the inversion and higher-order corrections. This is shown in Fig. 8, from which one can find that the error only becomes sizable when $|x|$ is small. This is expected because the relevant momentum scale is $x P^{z}$ such that higher-order corrections become large at small $x$.

There are more sophisticated methods to invert the factorization, such as using a recursion procedure. However, as we can see in Fig. 7, the systematic error caused by the matching procedure (thick-dashed-cyan line) is also smaller than those from the first two sources in most regions.

As shown in Fig. 7, one can find that the dominant uncertainties arise from the $p_{z}^{R}$ dependence and from FT. With $p_{z}^{R}=0$, the uncertainties from different projections, and inversion, and the matching are typically less than $10 \%$ except in the region with very small $|x|$. The uncertainty from
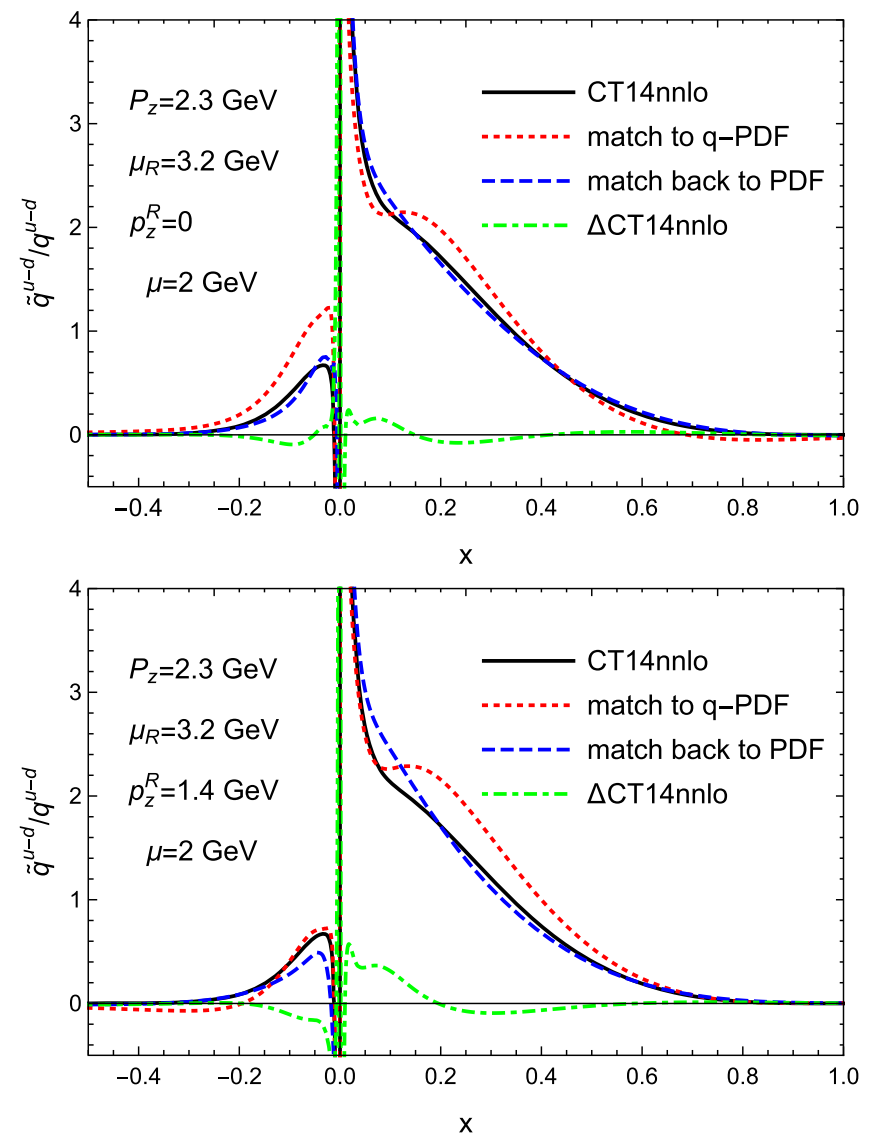

FIG. 8. Effects of the inversion matching formula using minimal projection. The solid-black, dotted-red, dotted-blue, and dot-dashed-green lines represent the CT14nnlo PDF, applying inverse matching from the CT14nnlo PDF [85] to the quasiPDF, applying matching again to get back to the PDF, the difference between the PDF with iterative matching and the original CT14nnlo PDF. The upper (lower) figure corresponds to $p_{z}^{R}=0 \mathrm{GeV}(1.4 \mathrm{GeV})$. These plots show that the method we used to invert the matching formula is less reliable for small $|x|$. The difference shown by the dot-dashed-green curve is taken into account in our systematic error. the $\mu_{R}$ dependence is even smaller. With $p_{z}^{R}=1.4 \mathrm{GeV}$, uncertainties from these three sources are getting larger in magnitude, but still smaller than those from the two major sources.

\section{FINAL RESULTS FOR PDF}

With the derivative method of FT and the matching using $p_{z}^{R}=0 \mathrm{GeV}$ and $\mu_{R}=3.2 \mathrm{GeV}$, we show the dependence on the nucleon boosted momentum in Fig. 9 with the statistical uncertainties. They are consistent with each other as we can expect from the consistency of the quasi-PDF matrix element results in Fig. 5.

Finally, we show our results for the PDF and a comparison with global analysis [85-87] in Fig. 10. As can be

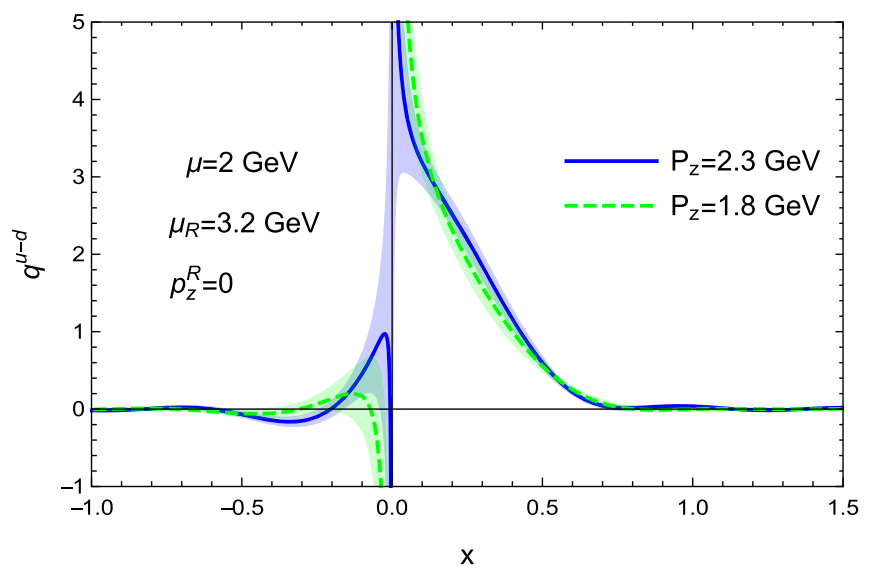

FIG. 9. Nucleon boost momentum dependence of the matched unpolarized isovector PDFs: the dotted-green and solid-blue lines correspond to the nucleon momentum $P_{z}$ being 1.8 and $2.3 \mathrm{GeV}$, respectively. The cutoff of Fourier transformation is chosen to be $z P^{z} \sim 12\left(z=15 a\right.$ for $P_{z}=1.8 \mathrm{GeV}$ and $z=12 a$ for $P_{z}=2.3 \mathrm{GeV}$ ).

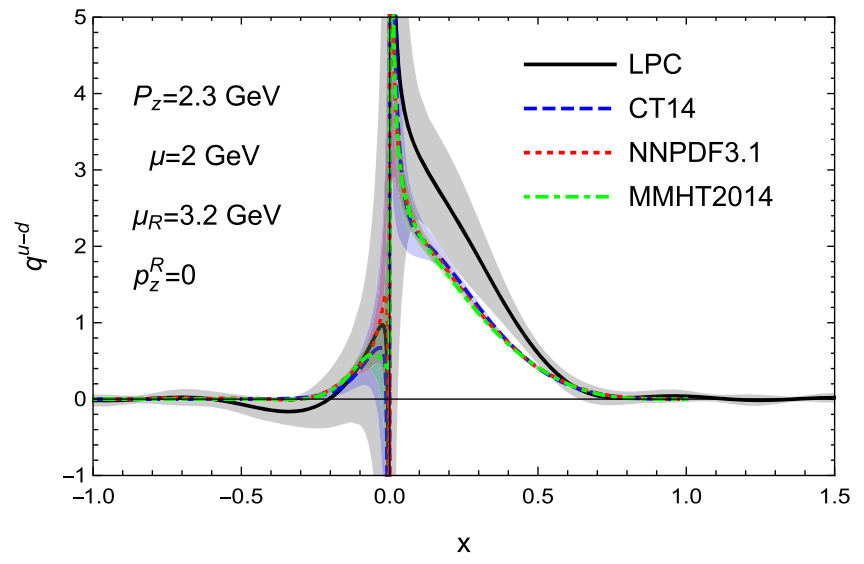

FIG. 10. Results for PDF at $\mu=2 \mathrm{GeV}$ calculated from the RI/MOM quasi-PDF at nucleon momentum $P_{z}=2.3 \mathrm{GeV}$ compared with CT14nnlo (90CL) [85], NNPDF3.1 (68CL) [86], and MMHT2014 (68CL) [87]. Our results agree with the global analysis within uncertainties. 
seen from the plot, our results show a reasonable agreement in the large- $x$ region, but in the small- $x$ region there exists a notable difference mainly due to the systematic uncertainties from the FT truncation method and also the $p_{z}^{R}$ dependence.

\section{SUMMARY}

In this paper, we have studied the quasi-PDF defined with $\gamma^{t}$ which is free from mixing at $\mathcal{O}\left(a^{0}\right)$. We have used $M_{\pi}=356 \mathrm{MeV}$ lattice data to demonstrate the matching procedure and show that the excited-state contamination is well under control. The one-loop matching coefficient has been calculated and we have discussed the sources of systematic errors as well as the choice of the projection in detail.

We have found that the systematic uncertainties from the FT truncation method and also the $p_{z}^{R}$ dependence are sizable. But those uncertainties from $\mu_{R}$, inversion of matching, and choice of projection are relatively minor with $p_{z}^{R}=0$. At the same time, the significant change from a quasi-PDF to a matched PDF suggests that higher-loop corrections are needed, as exhibited in Fig. 6.

Controlling systematic uncertainty from the excited state is very challenging since the relative uncertainty grows very fast when either source-sink separation $t_{\text {seq }}$ or nucleon momentum $P_{z}$ becomes large. The two-state fit with smaller separation provides a possibility to obtain a precise result in small $t_{\text {seq }}<1 \mathrm{fm}$ region, while for an accurate measurement at large separation using very high statistics, estimating the systematic uncertainty of such a fit is still needed.

Besides the uncertainties that we have studied, in the future we plan to investigate other systematics such as lattice discretization and finite volume effects [88] as well as higher-twist contributions that affect the small- $x$ result. The latter can be improved with larger nucleon momentum and estimated by extrapolating to infinite nucleon momentum.

Our final result for light-cone PDF agrees with the global analysis in the large- $x$ region, which gives an encouraging signal that LaMET may allow us to precisely access parton physics in the future.

\section{ACKNOWLEDGMENTS}

We thank the CLS Collaboration for sharing the lattices used to perform this study. The lattice QCD calculations were performed using the CHROMA software suite [82]. We thank Xiangdong Ji for the useful discussions. Y.-S. L. thanks Jun Gao and Shuai Zhao for the useful discussions, and Y.-B. Y. thanks Huey-Wen Lin for the discussion on part of the simulation setup. The numerical calculation is supported by the Center for HPC of Shanghai Jiao Tong University, HPC Cluster of ITP-CAS, Jiangsu Key Lab for NSLSCS, and Strategic Priority Research Program of Chinese Academy of Sciences Grant No. XDC01040100. J.-W. C. is partly supported by the Ministry of Science and Technology, Taiwan, under Grant No. 105-2112-M-002017-MY3 and the Kenda Foundation. L. J. is supported by the Department of Energy, Laboratory Directed Research and Development (LDRD) funding of BNL, under Contract No. DE-EC0012704. Y.-S. L. is supported by the Science and Technology Commission of Shanghai Municipality under Grant No. 16DZ2260200 and the National Natural Science Foundation of China under Grant No. 11905126. P. S. is supported by the Natural Science Foundation of China under Grant No. 11975127. W. W. is supported in part by the Natural Science Foundation of China under Grants No. 11575110, No. 11735010, and No. 11911530088, and by the Natural Science Foundation of Shanghai under Grant No. 15DZ2272100. Y.-B. Y. is partly supported by the U. S. National Science Foundation under Grant No. PHY 1653405 "CAREER: Constraining parton distribution functions for new-physics searches" and the CAS Pioneer Hundred Talents Program. J.-H.Z. is supported by the Natural Science Foundation of China under Grant No. 11975051. A. S., M. S., and J.-H. Z. are supported by SFB/TRR-55 grant "Hadron physics from lattice QCD." Q.A.Z. is supported by the China Postdoctoral Science Foundation and the National Postdoctoral Program for Innovative Talents (Grant No. BX20190207). Y.Z. is supported in part by the U.S. Department of Energy, Office of Science, Office of Nuclear Physics, under Grants No. DE-SC0011090 and No. DE-SC0012704 and within the framework of the TMD Topical Collaboration.

\section{APPENDIX}

\section{One-loop quasi-PDF with $\gamma^{\alpha}$ in general covariant gauge}

The gluon propagator in the general covariant gauge is

$$
i D_{\tau}^{\mu \nu}(k)=-\frac{i}{k^{2}}\left[g^{\mu \nu}-(1-\tau) \frac{k^{\mu} k^{\nu}}{k^{2}}\right]
$$

For general $\Gamma=\gamma^{\alpha}$, the one-loop result can be expressed as

$$
\tilde{q}^{(1)}(x, p, \rho)=\operatorname{Tr}\left[\left(\left[\tilde{f}_{\alpha}(x, \rho)\right]_{+} \gamma^{\alpha}+\left[\tilde{f}_{z}(x, \rho)\right]_{+} \frac{p_{\alpha}}{p_{z}} \gamma^{z}+\left[\tilde{f}_{p}(x, \rho)\right]_{+} \frac{p_{\alpha} \not p}{p^{2}}\right) \mathcal{P}\right],
$$

where 


$$
\begin{aligned}
& \tilde{f}_{\alpha}(x, \rho)=\frac{\alpha_{s} C_{F}}{2 \pi}\left\{\begin{array}{lc}
\frac{x-\rho}{(1-x)(1-\rho)}+\frac{2 x(2-x)-\rho(1+x)}{2(1-x)(1-\rho)^{3 / 2}} \ln \frac{2 x-1+\sqrt{1-\rho}}{2 x-1-\sqrt{1-\rho}} & x>1 \\
\frac{-3 x+2 x^{2}+\rho}{(1-x)(1-\rho)}+\frac{2 x(2-x)-\rho(1+x)}{2(1-x)(1-\rho)^{3 / 2}} \ln \frac{1+\sqrt{1-\rho}}{1-\sqrt{1-\rho}} & 0<x<1 \\
-\frac{x-\rho}{(1-x)(1-\rho)}-\frac{2 x(2-x)-\rho(1+x)}{2(1-x)(1-\rho)^{3 / 2}} \ln \frac{2 x-1+\sqrt{1-\rho}}{2 x-1-\sqrt{1-\rho}} & x<0
\end{array}\right. \\
& +\frac{\alpha_{s} C_{F}}{2 \pi}(1-\tau) \begin{cases}\frac{\rho\left(-3 x+2 x^{2}+\rho\right)}{2(1-x)(1-\rho)\left(4 x-4 x^{2}-\rho\right)}+\frac{-\rho}{4(1-\rho)^{3 / 2}} \ln \frac{2 x-1+\sqrt{1-\rho}}{2 x-1-\sqrt{1-\rho}} & x>1 \\
\frac{-x+\rho}{2(1-x)(1-\rho)}+\frac{-\rho}{4(1-\rho)^{3 / 2}} \ln \frac{1+\sqrt{1-\rho}}{1-\sqrt{1-\rho}} & 0<x<1, \\
-\frac{\rho\left(-3 x+2 x^{2}+\rho\right)}{2(1-x)(1-\rho)\left(4 x-4 x^{2}-\rho\right)}-\frac{-\rho}{4(1-\rho)^{3 / 2}} \ln \frac{2 x-1+\sqrt{1-\rho}}{2 x-1-\sqrt{1-\rho}} & x<0\end{cases} \\
& \tilde{f}_{z}(x, \rho)=\frac{\alpha_{s} C_{F}}{2 \pi} \begin{cases}\frac{-2 \rho\left(1-7 x+6 x^{2}\right)-\rho^{2}(1+2 x)}{(1-\rho)^{2}\left(4 x-4 x^{2}-\rho\right)} g_{z \alpha}+\frac{4 x\left(1-3 x+2 x^{2}\right)-\rho\left(2-11 x+12 x^{2}-4 x^{3}\right)-\rho^{2}}{(1-x)(1-\rho)^{2}\left(4 x-4 x^{2}-\rho\right)} & x>1 \\
+\left[\frac{\rho(4-6 x-\rho)}{2(1-\rho)^{5 / 2}} g_{z \alpha}+\frac{2-4 x+4 x^{2}-5 x \rho+2 x^{2} \rho+\rho^{2}}{2(1-x)(1-\rho)^{5 / 2}}\right] \ln \frac{2 x-1+\sqrt{1-\rho}}{2 x-1-\sqrt{1-\rho}} & 0<x<1 \\
\frac{-2+2 x-\rho(1-4 x)}{(1-\rho)^{2}} g_{z \alpha}+\frac{(-1+2 x)(2-3 x+\rho)}{(1-x)(1-\rho)^{2}} & \\
+\left[\frac{\rho(4-6 x-\rho)}{2(1-\rho)^{5 / 2}} g_{z \alpha}+\frac{2-4 x+4 x^{2}-5 x \rho+2 x^{2} \rho+\rho^{2}}{2(1-x)(1-\rho)^{5 / 2}}\right] \ln \frac{1+\sqrt{1-\rho}}{1-\sqrt{1-\rho}} & x<0 \\
-\frac{-2 \rho\left(1-7 x+6 x^{2}\right)-\rho^{2}(1+2 x)}{(1-\rho)^{2}\left(4 x-4 x^{2}-\rho\right)} g_{z \alpha}-\frac{4 x\left(1-3 x+2 x^{2}\right)-\rho\left(2-11 x+12 x^{2}-4 x^{3}\right)-\rho^{2}}{(1-x)(1-\rho)^{2}\left(4 x-4 x^{2}-\rho\right)} & \\
-\left[\frac{\rho(4-6 x-\rho)}{2(1-\rho)^{5 / 2}} g_{z \alpha}+\frac{2-4 x+4 x^{2}-5 x \rho+2 x^{2} \rho+\rho^{2}}{2(1-x)(1-\rho)^{5 / 2}}\right] \ln \frac{2 x-1+\sqrt{1-\rho}}{2 x-1-\sqrt{1-\rho}} & \end{cases} \\
& +\frac{\alpha_{s} C_{F}}{2 \pi}(1-\tau) \begin{cases}\frac{\rho(1-2 x)\left[-4 x(1-x)(2+\rho)+3 \rho^{2}\right]}{2(1-\rho)^{2}\left(4 x-4 x^{2}-\rho\right)^{2}} g_{z \alpha}+\frac{\rho\left[-4 x\left(2-9 x+6 x^{2}\right)+\rho(1-10 x+2 \rho)\right]}{2(1-\rho)^{2}\left(4 x-4 x^{2}-\rho\right)^{2}} & x>1 \\
+\frac{\left.\rho\left[(2+\rho) g_{z \alpha}+3\right)\right]}{4(1-\rho)^{5 / 2}} \ln \frac{2 x-1+\sqrt{1-\rho}}{2 x-1-\sqrt{1-\rho}} & 0<x<1, \\
\frac{-3 \rho g_{z \alpha}-1-2 \rho}{2(1-\rho)^{2}}+\frac{\left.\rho\left[(2+\rho) g_{z \alpha}+3\right)\right]}{4(1-\rho)^{5 / 2}} \ln \frac{1+\sqrt{1-\rho}}{1-\sqrt{1-\rho}} & x<0 \\
-\frac{\rho(1-2 x)\left[-4 x(1-x)(2+\rho)+3 \rho^{2}\right]}{2(1-\rho)^{2}\left(4 x-4 x^{2}-\rho\right)^{2}} g_{z \alpha}-\frac{\rho\left[-4 x\left(2-9 x+6 x^{2}\right)+\rho(1-10 x+2 \rho)\right]}{2(1-\rho)^{2}\left(4 x-4 x^{2}-\rho\right)^{2}} & \\
-\frac{\left.\rho\left[(2+\rho) g_{z \alpha}+3\right)\right]}{4(1-\rho)^{5 / 2}} \ln \frac{2 x-1+\sqrt{1-\rho}}{2 x-1-\sqrt{1-\rho}} & \end{cases}
\end{aligned}
$$

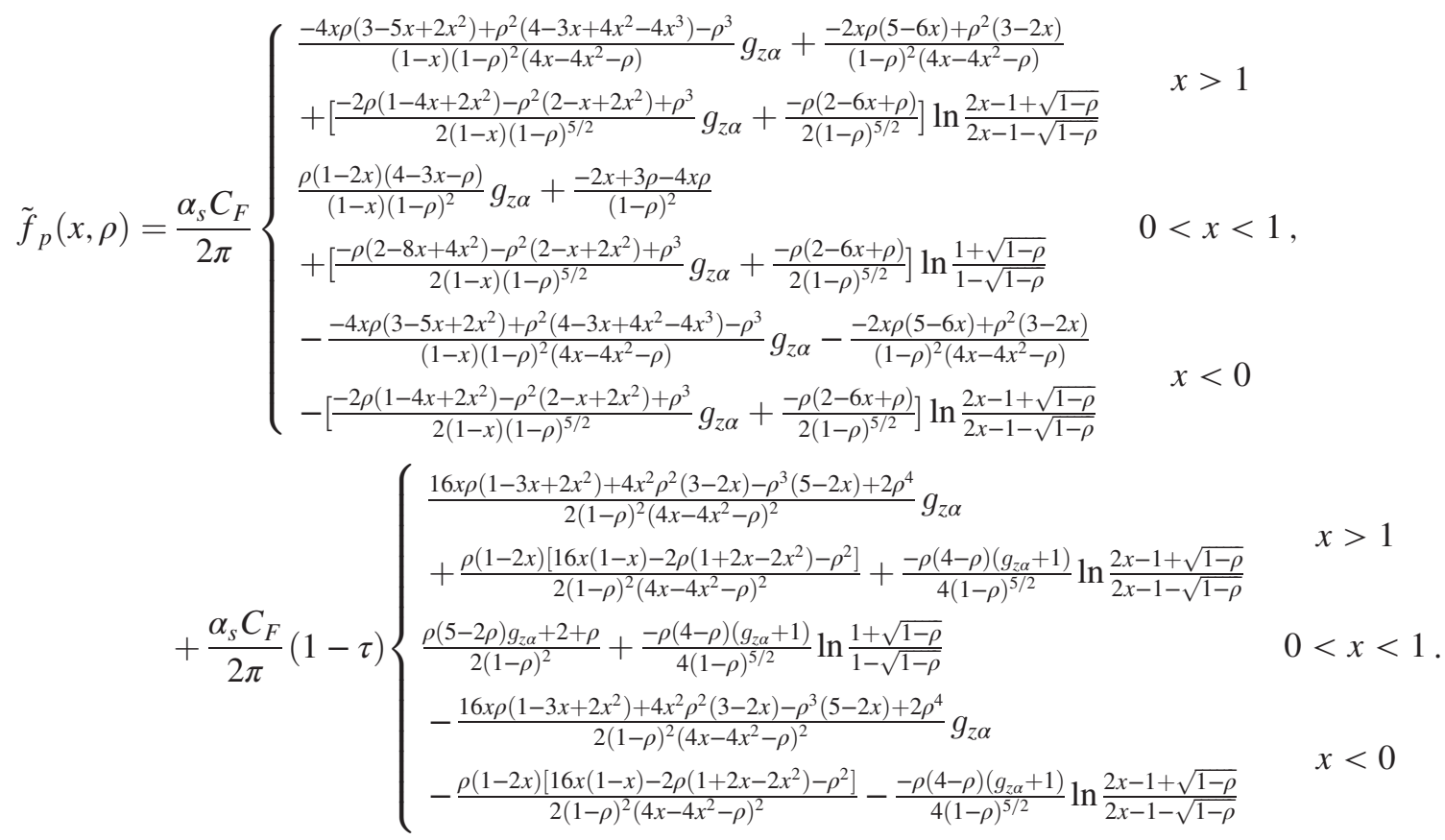




\section{One-loop quasi-PDF with $\Gamma=\gamma^{z}$ in Landau gauge}

For $\Gamma=\gamma^{z}$, we can obtain the matching coefficient Eq. (31) using the general formula with a similar definition of the minimal and $\not p$ projections in Sec. II B. The bare matching coefficients are

$$
f_{1, m p}\left(x, \frac{p_{z}}{\mu}\right)=f_{1, \not p}\left(x, \frac{p_{z}}{\mu}\right)=\frac{\alpha_{s} C_{F}}{2 \pi}\left\{\begin{array}{lc}
\frac{1+x^{2}}{1-x} \ln \frac{x}{x-1}+1 & x>1 \\
\frac{1+x^{2}}{1-x} \ln \frac{4 x(1-x) p_{z}^{2}}{\mu^{2}}+\frac{2-5 x+x^{2}}{1-x} & 0<x<1, \\
-\frac{1+x^{2}}{1-x} \ln \frac{x}{x-1}-1 & x<0
\end{array}\right.
$$

and the corresponding counterterms are

$$
\begin{aligned}
& f_{2, m p}(x, r)=\frac{\alpha_{s} C_{F}}{2 \pi}\left\{\begin{array}{lc}
\frac{3 r-(1-2 x)^{2}}{2(r-1)(1-x)}-\frac{4 x^{2}\left(2-3 r+2 x+4 r x-12 x^{2}+8 x^{3}\right)}{(r-1)\left(r-4 x+4 x^{2}\right)^{2}}+\frac{2-3 r+2 x^{2}}{(r-1)^{3 / 2}(x-1)} \tan ^{-1} \frac{\sqrt{r-1}}{2 x-1} & x>1 \\
\frac{1-3 r+4 x^{2}}{2(r-1)(1-x)}+\frac{-2+3 r-2 x^{2}}{(r-1)^{3 / 2}(1-x)} \tan ^{-1} \sqrt{r-1} & 0<x<1, \\
-\frac{3 r-(1-2 x)^{2}}{2(r-1)(1-x)}+\frac{4 x^{2}\left(2-3 r+2 x+4 r x-12 x^{2}+8 x^{3}\right)}{(r-1)\left(r-4 x+4 x^{2}\right)^{2}}-\frac{2-3 r+2 x^{2}}{(r-1)^{3 / 2}(x-1)} \tan ^{-1} \frac{\sqrt{r-1}}{2 x-1} & x<0
\end{array}\right. \\
& f_{2, \not p}(x, r)=\frac{\alpha_{s} C_{F}}{2 \pi}\left\{\begin{array}{lc}
1+\frac{r}{2} \frac{r(3-4 x)-8 x(x-1)^{2}}{(x-1)\left(r-4 x+4 x^{2}\right)^{2}}+\frac{-2+r-2 x^{2}}{\sqrt{r-1}(x-1)} \tan ^{-1} \frac{\sqrt{r-1}}{2 x-1} & x>1 \\
\frac{1-6 x}{2(1-x)}+\frac{2-r+2 x^{2}}{\sqrt{r-1}(1-x)} \tan ^{-1} \sqrt{r-1} & 0<x<1 . \\
-1-\frac{r}{2} \frac{r(3-4 x)-8 x(x-1)^{2}}{(x-1)\left(r-4 x+4 x^{2}\right)^{2}}-\frac{-2+r-2 x^{2}}{\sqrt{r-1}(x-1)} \tan ^{-1} \frac{\sqrt{r-1}}{2 x-1} & x<0
\end{array}\right.
\end{aligned}
$$

The result was also calculated in Ref. [11]. Although the matching coefficient with $\Gamma=\gamma^{z}$ is not useful for an isovector unpolarized PDF because it suffers from operator mixing in renormalization procedure, it can be used for an isovector helicity PDF due to different symmetry properties.

[1] J. Butterworth et al., PDF4LHC recommendations for LHC Run II, J. Phys. G 43, 023001 (2016).

[2] S. Alekhin, J. Blümlein, S. Moch, and R. Placakyte, Parton distribution functions, $\alpha_{s}$, and heavy-quark masses for LHC Run II, Phys. Rev. D 96, 014011 (2017).

[3] J. Gao, L. Harland-Lang, and J. Rojo, The structure of the proton in the LHC precision era, Phys. Rep. 742, 1 (2018).

[4] J. Collins, Foundations of Perturbative QCD, Cambridge Monographs on Particle Physics, Nuclear Physics and Cosmology Vol. 32 (Cambridge University Press, Cambridge, England, 2011), p. 1.

[5] G. Martinelli and C. T. Sachrajda, Pion structure functions from lattice QCD, Phys. Lett. B 196, 184 (1987).

[6] G. Martinelli and C. T. Sachrajda, The quark distribution amplitude of the proton: A lattice computation of the lowest two moments, Phys. Lett. B 217, 319 (1989).

[7] W. Detmold, W. Melnitchouk, and A. W. Thomas, Parton distributions from lattice QCD, Eur. Phys. J. direct E 3, 1 (2001).

[8] D. Dolgov et al. (LHPC and TXL Collaborations), Moments of nucleon light cone quark distributions calculated in full Lattice QCD, Phys. Rev. D 66, 034506 (2002).

[9] X. Ji, Parton Physics on a Euclidean Lattice, Phys. Rev. Lett. 110, 262002 (2013).
[10] X. Ji, Parton physics from large-momentum effective field theory, Sci. China Phys. Mech. Astron. 57, 1407 (2014).

[11] I. W. Stewart and Y. Zhao, Matching the quasiparton distribution in a momentum subtraction scheme, Phys. Rev. D 97, 054512 (2018).

[12] T. Izubuchi, X. Ji, L. Jin, I. W. Stewart, and Y. Zhao, Factorization theorem relating Euclidean and light-cone parton distributions, Phys. Rev. D 98, 056004 (2018).

[13] Y.Q. Ma and J.W. Qiu, Extracting parton distribution functions from lattice QCD calculations, Phys. Rev. D 98, 074021 (2018).

[14] Y. Q. Ma and J. W. Qiu, Exploring Partonic Structure of Hadrons Using ab initio Lattice QCD Calculations, Phys. Rev. Lett. 120, 022003 (2018).

[15] H. W. Lin, J. W. Chen, S. D. Cohen, and X. Ji, Flavor structure of the nucleon sea from lattice QCD, Phys. Rev. D 91, 054510 (2015).

[16] C. Alexandrou, K. Cichy, V. Drach, E. Garcia-Ramos, K. Hadjiyiannakou, K. Jansen, F. Steffens, and C. Wiese, Lattice calculation of parton distributions, Phys. Rev. D 92, 014502 (2015).

[17] J. W. Chen, S. D. Cohen, X. Ji, H. W. Lin, and J. H. Zhang, Nucleon helicity and transversity parton distributions from lattice QCD, Nucl. Phys. B911, 246 (2016). 
[18] C. Alexandrou, K. Cichy, M. Constantinou, K. Hadjiyiannakou, K. Jansen, F. Steffens, and C. Wiese, Updated lattice results for parton distributions, Phys. Rev. D 96, 014513 (2017).

[19] J. H. Zhang, J. W. Chen, L. Jin, H. W. Lin, A. Schäfer, and Y. Zhao, First direct lattice-QCD calculation of the $x$-dependence of the pion parton distribution function, Phys. Rev. D 100, 034505 (2019).

[20] C. Alexandrou, K. Cichy, M. Constantinou, K. Jansen, A. Scapellato, and F. Steffens, Transversity parton distribution functions from lattice QCD, Phys. Rev. D 98, 091503 (2018)

[21] J. H. Zhang, J. W. Chen, X. Ji, L. Jin, and H. W. Lin, Pion distribution amplitude from lattice QCD, Phys. Rev. D 95, 094514 (2017).

[22] J. H. Zhang, J.-W. Chen, L. Jin, H.-W. Lin, A. Schäfer, P. Sun, Y.-B. Yang, J.-H. Zhang, and Y. Zhao (LP3 Collaboration), Kaon distribution amplitude from lattice QCD and the flavor SU(3) symmetry, Nucl. Phys. B939, 429 (2019).

[23] X. Xiong, X. Ji, J. H. Zhang, and Y. Zhao, One-loop matching for parton distributions: Nonsinglet case, Phys. Rev. D 90, 014051 (2014).

[24] X. Ji, A. Schäfer, X. Xiong, and J. H. Zhang, One-loop matching for generalized parton distributions, Phys. Rev. D 92, 014039 (2015).

[25] X. Xiong and J. H. Zhang, One-loop matching for transversity generalized parton distribution, Phys. Rev. D 92, 054037 (2015).

[26] H. N. Li, Nondipolar Wilson links for quasiparton distribution functions, Phys. Rev. D 94, 074036 (2016).

[27] G. C. Rossi and M. Testa, Note on lattice regularization and equal-time correlators for parton distribution functions, Phys. Rev. D 96, 014507 (2017).

[28] G. Rossi and M. Testa, Euclidean versus Minkowski short distance, Phys. Rev. D 98, 054028 (2018).

[29] X. Ji and J.H. Zhang, Renormalization of quasiparton distribution, Phys. Rev. D 92, 034006 (2015).

[30] T. Ishikawa, Y. Q. Ma, J. W. Qiu, and S. Yoshida, Practical quasi parton distribution functions, arXiv:1609.02018.

[31] J. W. Chen, X. Ji, and J. H. Zhang, Improved quasi parton distribution through Wilson line renormalization, Nucl. Phys. B915, 1 (2017).

[32] X. Xiong, T. Luu, and U. G. Meißner, Quasi-parton distribution function in lattice perturbation theory, arXiv:1705.00246.

[33] M. Constantinou and H. Panagopoulos, Perturbative renormalization of quasi-parton distribution functions, Phys. Rev. D 96, 054506 (2017).

[34] X. Ji, J. H. Zhang, and Y. Zhao, Renormalization in Large Momentum Effective Theory of Parton Physics, Phys. Rev. Lett. 120, 112001 (2018).

[35] T. Ishikawa, Y. Q. Ma, J. W. Qiu, and S. Yoshida, Renormalizability of quasiparton distribution functions, Phys. Rev. D 96, 094019 (2017).

[36] J. Green, K. Jansen, and F. Steffens, Nonperturbative Renormalization of Nonlocal Quark Bilinears for Parton Quasidistribution Functions on the Lattice Using an Auxiliary Field, Phys. Rev. Lett. 121, 022004 (2018).

[37] G. Spanoudes and H. Panagopoulos, Renormalization of Wilson-line operators in the presence of nonzero quark masses, Phys. Rev. D 98, 014509 (2018).
[38] C. Alexandrou, K. Cichy, M. Constantinou, K. Hadjiyiannakou, K. Jansen, H. Panagopoulos, and F. Steffens, A complete nonperturbative renormalization prescription for quasi-PDFs, Nucl. Phys. B923, 394 (2017).

[39] J. W. Chen, T. Ishikawa, L. Jin, H. W. Lin, Y. B. Yang, J. H. Zhang, and Y. Zhao, Parton distribution function with nonperturbative renormalization from lattice QCD, Phys. Rev. D 97, 014505 (2018).

[40] G. Martinelli, C. Pittori, C. T. Sachrajda, M. Testa, and A. Vladikas, A general method for nonperturbative renormalization of lattice operators, Nucl. Phys. B445, 81 (1995).

[41] H. W. Lin, J.-W. Chen, T. Ishikawa, and J.-H. Zhang (LP3 Collaboration), Improved parton distribution functions at the physical pion mass, Phys. Rev. D 98, 054504 (2018).

[42] C. Alexandrou, K. Cichy, M. Constantinou, K. Jansen, A. Scapellato, and F. Steffens, Light-Cone Parton Distribution Functions from Lattice QCD, Phys. Rev. Lett. 121, 112001 (2018).

[43] J. W. Chen, L. Jin, H. W. Lin, Y. S. Liu, Y. B. Yang, J. H. Zhang, and Y. Zhao, Lattice calculation of parton distribution function from LaMET at physical pion mass with large nucleon momentum, arXiv:1803.04393.

[44] J. W. Chen, T. Ishikawa, L. Jin, H.-W. Lin, J.-H. Zhang, and Y. Zhao (LP3 Collaboration), Symmetry properties of nonlocal quark bilinear operators on a lattice, Chin. Phys. C 43, 103101 (2019).

[45] T. Ishikawa, L. Jin, H. W. Lin, A. Schäfer, Y. B. Yang, J. H. Zhang, and Y. Zhao, Gaussian-weighted parton quasidistribution [Lattice Parton Physics Project $\left(\mathrm{LP}^{3}\right)$ ], Sci. China Phys. Mech. Astron. 62, 991021 (2019).

[46] X. Ji, P. Sun, X. Xiong, and F. Yuan, Soft factor subtraction and transverse momentum dependent parton distributions on the lattice, Phys. Rev. D 91, 074009 (2015).

[47] X. Ji, L. C. Jin, F. Yuan, J. H. Zhang, and Y. Zhao, Transverse momentum dependent parton quasidistributions, Phys. Rev. D 99, 114006 (2019).

[48] M. A. Ebert, I. W. Stewart, and Y. Zhao, Determining the nonperturbative Collins-Soper kernel from lattice QCD, Phys. Rev. D 99, 034505 (2019).

[49] M. A. Ebert, I. W. Stewart, and Y. Zhao, Towards quasitransverse momentum dependent PDFs computable on the lattice, J. High Energy Phys. 09 (2019) 037.

[50] M. A. Ebert, I. W. Stewart, and Y. Zhao, Renormalization and matching for the Collins-Soper kernel from lattice QCD, arXiv:1910.08569.

[51] X. Ji, Y. Liu, and Y. S. Liu, QCD soft function from largemomentum effective theory on lattice, arXiv:1910.11415.

[52] P. Shanahan, M. Wagman, and Y. Zhao, Nonperturbative renormalization of staple-shaped Wilson line operators in lattice QCD, arXiv:1911.00800.

[53] X. Ji, Y. Liu, and Y.S. Liu, Transverse-momentumdependent PDFs from large-momentum effective theory, arXiv:1911.03840.

[54] W. Wang, S. Zhao, and R. Zhu, Gluon quasidistribution function at one loop, Eur. Phys. J. C 78, 147 (2018).

[55] W. Wang and S. Zhao, On the power divergence in quasi gluon distribution function, J. High Energy Phys. 05 (2018) 142.

[56] Z. Y. Fan, Y. B. Yang, A. Anthony, H. W. Lin, and K. F. Liu, Gluon Quasi-Parton-Distribution Functions from Lattice QCD, Phys. Rev. Lett. 121, 242001 (2018). 
[57] J. H. Zhang, X. Ji, A. Schäfer, W. Wang, and S. Zhao, Accessing Gluon Parton Distributions in Large Momentum Effective Theory, Phys. Rev. Lett. 122, 142001 (2019).

[58] Z. Y. Li, Y. Q. Ma, and J. W. Qiu, Multiplicative Renormalizability of Operators Defining Quasiparton Distributions, Phys. Rev. Lett. 122, 062002 (2019).

[59] W. Wang, J. H. Zhang, S. Zhao, and R. Zhu, Complete matching for quasidistribution functions in large momentum effective theory, Phys. Rev. D 100, 074509 (2019).

[60] C. Monahan and K. Orginos, Quasi parton distributions and the gradient flow, J. High Energy Phys. 03 (2017) 116.

[61] C. Monahan, Smeared quasidistributions in perturbation theory, Phys. Rev. D 97, 054507 (2018).

[62] A. V. Radyushkin, Quasi-parton distribution functions, momentum distributions, and pseudo-parton distribution functions, Phys. Rev. D 96, 034025 (2017).

[63] K. Orginos, A. Radyushkin, J. Karpie, and S. Zafeiropoulos, Lattice QCD exploration of parton pseudo-distribution functions, Phys. Rev. D 96, 094503 (2017).

[64] J. Karpie, K. Orginos, A. Radyushkin, and S. Zafeiropoulos, Parton distribution functions on the lattice and in the continuum, EPJ Web Conf. 175, 06032 (2018).

[65] J. H. Zhang, J. W. Chen, and C. Monahan, Parton distribution functions from reduced Ioffe-time distributions, Phys. Rev. D 97, 074508 (2018).

[66] K. F. Liu and S. J. Dong, Origin of difference between anti-d and anti-u partons in the nucleon, Phys. Rev. Lett. 72, 1790 (1994).

[67] J. Liang, K. F. Liu, and Y. B. Yang, Lattice calculation of hadronic tensor of the nucleon, EPJ Web Conf. 175, 14014 (2018).

[68] W. Detmold and C. J. D. Lin, Deep-inelastic scattering and the operator product expansion in lattice QCD, Phys. Rev. D 73, 014501 (2006).

[69] V. Braun and D. Müller, Exclusive processes in position space and the pion distribution amplitude, Eur. Phys. J. C 55, 349 (2008).

[70] A. J. Chambers, R. Horsley, Y. Nakamura, H. Perlt, P. E. L. Rakow, G. Schierholz, A. Schiller, K. Somfleth, R. D. Young, and J. M. Zanotti, Nucleon Structure Functions from Operator Product Expansion on the Lattice, Phys. Rev. Lett. 118, 242001 (2017).

[71] G. S. Bali, V. M. Braun, B. Gläßle, M. Göckeler, M. Gruber, F. Hutzler, P. Korcyl, A. Schäfer, P. Wein, and J.-H. Zhang, Pion distribution amplitude from Euclidean correlation functions: Exploring universality and higher-twist effects, Phys. Rev. D 98, 094507 (2018).

[72] G. S. Bali et al. (RQCD Collaboration), Light-cone distribution amplitudes of octet baryons from lattice QCD, Eur. Phys. J. A 55, 116 (2019).

[73] C. E. Carlson and M. Freid, Lattice corrections to the quark quasidistribution at one-loop, Phys. Rev. D 95, 094504 (2017).
[74] X. Ji, J. H. Zhang, and Y. Zhao, More on large-momentum effective theory approach to parton physics, Nucl. Phys. B924, 366 (2017).

[75] R. A. Briceño, M. T. Hansen, and C. J. Monahan, Role of the Euclidean signature in lattice calculations of quasidistributions and other nonlocal matrix elements, Phys. Rev. D 96, 014502 (2017).

[76] M. Lüscher and S. Schäfer, Lattice QCD without topology barriers, J. High Energy Phys. 07 (2011) 036.

[77] M. Bruno et al., Simulation of QCD with $\mathrm{N}_{f}=2+1$ flavors of non-perturbatively improved Wilson fermions, J. High Energy Phys. 02 (2015) 043.

[78] M. Falcioni et al., Again on SU(3) glueball mass, Nucl. Phys. B251, 624 (1985); M. Albanese et al., Glueball masses and string tension in lattice QCD, Phys. Lett. B 192, 163 (1987).

[79] G. S. Bali, B. Lang, B. U. Musch, and A. Schäfer, Novel quark smearing for hadrons with high momenta in lattice QCD, Phys. Rev. D 93, 094515 (2016).

[80] R. Babich, J. Brannick, R. C. Brower, M. A. Clark, T. A. Manteuffel, S. F. McCormick, J. C. Osborn, and C. Rebbi, Adaptive multigrid algorithm for the lattice Wilson-Dirac operator, Phys. Rev. Lett. 105, 201602 (2010).

[81] J. C. Osborn, R. Babich, J. Brannick, R. C. Brower, M. A. Clark, S. D. Cohen, and C. Rebbi, Multigrid solver for clover fermions, Proc. Sci., Lattice2010 (2010) 037 [arXiv: 1011.2775].

[82] R. G. Edwards, and B. Joó (SciDAC, LHPC, and UKQCD Collaborations), The CHROMA software system for lattice QCD, Nucl. Phys. B, Proc. Suppl. 140, 832 (2005).

[83] T. Bhattacharya, S. D. Cohen, R. Gupta, A. Joseph, H. W. Lin, and B. Yoon, Nucleon charges and electromagnetic form factors from $2+1+1$-flavor lattice QCD, Phys. Rev. D 89, 094502 (2014).

[84] J. Karpie, K. Orginos, A. Rothkopf, and S. Zafeiropoulos, Reconstructing parton distribution functions from Ioffe time data: From Bayesian methods to neural networks, J. High Energy Phys. 04 (2019) 057.

[85] S. Dulat, T.-J. Hou, J. Gao, M. Guzzi, J. Huston, P. Nadolsky, J. Pumplin, C. Schmidt, D. Stump, and C.-P. Yuan, New parton distribution functions from a global analysis of quantum chromodynamics, Phys. Rev. D 93, 033006 (2016).

[86] R. D. Ball et al. (NNPDF Collaboration), Parton distributions from high-precision collider data, Eur. Phys. J. C 77, 663 (2017).

[87] L. A. Harland-Lang, A. D. Martin, P. Motylinski, and R. S. Thorne, Parton distributions in the LHC era: MMHT 2014 PDFs, Eur. Phys. J. C 75, 204 (2015).

[88] H. W. Lin and R. Zhang, Lattice finite-volume dependence of the nucleon parton distributions, Phys. Rev. D 100, 074502 (2019). 